\title{
SUSTENTABILIDAD DE DOS SISTEMAS DE PRODUCCIÓN DE ARROZ, UNO EN CONDICIONES DE SALINIDAD EN LA ZONA DE YAGUACHI Y OTRO EN CONDICIONES NORMALES EN EL SISTEMA DE RIEGO Y DRENAJE BABAHOYO, ECUADOR
}

\section{SUSTAINABILITY OF TWO RICE PRODUCTION SYSTEMS, ONE UNDER SALINITY CONDITIONS IN THE YAGUACHI AREA AND THE OTHER UNDER NORMAL CONDITIONS IN THE BABAHOYO IRRIGATION AND DRAINAGE SYSTEM, ECUADOR}

\author{
Fernando Javier Cobos Mora ${ }^{1}$, Luz Rayda Gómez Pando ${ }^{2}$, Walter Oswaldo Reyes Borja ${ }^{3}$ y \\ Reina Concepción Medina Litardo ${ }^{4}$
}

\begin{abstract}
Resumen
Se realizó un estudio en las provincias de Guayas y Los Ríos (Ecuador), con el objetivo de caracterizar los sistemas de producción arrocero; uno en condiciones de salinidad en Yaguachi y otro sin problemas de salinización en el Cantón Babahoyo. Se elaboraron indicadores económicos, sociales y ecológicos, adecuados a los sistemas arroceros bajo estudio para determinar el nivel de sustentabilidad. La evaluación, mediante indicadores, permitió detectar de forma objetiva puntos críticos en estas localidades que afectan la sustentabilidad. En el aspecto ambiental, el indicador salinidad, fue calificado de nivel medio a alto riesgo, el indicador aplicación de agroquímicos, caracterizado por el uso intensivo de plaguicidas y fertilizantes es el que afecta más negativamente la sustentabilidad, especialmente, del sistema arrocero Yaguachi. En la dimensión económica, el indicador sistema de monocultivo que genera pérdidas de ingresos adicionales y la falta de diversidad de productos utilizados para la venta y la alimentación afectan en forma negativa a los dos sistemas de producción arroceros. En el aspecto social, los indicadores que contribuyen a una mayor sustentabilidad fueron la calidad de vivienda, grado de satisfacción y relaciones con otros miembros de la comunidad. Los Índices de Sustentabilidad General para los dos sistemas de producción arrocero en Yaguachi y Babahoyo, fueron 1.92 y 2.12, respectivamente.

Palabras clave: cultivo de arroz, salinidad del suelo, salinidad del agua, sustentabilidad de áreas agrícolas.
\end{abstract}

\begin{abstract}
A study was carried out in the provinces of Guayas and Los Ríos (Ecuador), with the objective of characterizing rice production systems; one under salinity conditions in Yaguachi and the other without salinization problems in the Babahoyo canton. Economic, social and ecological indicators were developed, suitable for the rice systems under study to determine the level of sustainability. The evaluation through indicators allowed to objectively detect critical points in these localities that affect sustainability. In the environmental aspect, the salinity indicator, classified as medium to high risk, and the indicator for the application of agrochemicals, characterized by the intensive use of pesticides and fertilizers, are those that most negatively affect the sustainability, especially, of the rice system in Yaguachi. In the economic dimension, the monoculture system indicator that generates additional income losses and the lack of diversity of products used for sale and food negatively affect the two rice production systems. In the social aspect, the indicators that contribute to greater sustainability were the quality of housing, degree of satisfaction and relationships with other members of the community. The General Sustainability Indices for the two rice production systems in Yaguachi and Babahoyo were 1.92 and 2.12, respectively.
\end{abstract}

Key words: rice cultivation, soil salinity, water salinity, sustainability of agricultural areas.

\section{Introducción}

Uno de los principales cereales básicos utilizados para la alimentación humana es el arroz (Oryza sativa L.), para el año 2018 se estima a nivel mundial una producción de 510.6 millones de toneladas (Mt) y una demanda de 505 Mt (FAOSTAT, 2018). En el año 2019, en el Ecuador, se registraron 261770 hectáreas de arroz, de las cuales el $67.38 \%$ estuvieron en la
Provincia de Guayas, $25.68 \%$ en Los Ríos y $2.08 \%$ en Loja. Los cantones con mayor producción son Daule (Guayas) y Babahoyo (Los Ríos) (INEC, 2020).

El arroz es cultivado en diferentes tipos de suelos, ambientes, y el éxito o fracaso depende del uso de tecnología y variedades apropiadas para asegurar rendimientos económicos; más aún, en ambientes desfavorables, los cuales se van incrementando por el 
Enero - Julio 2021

cambio climático y las malas prácticas agrícolas (Cobos et al., 2021).

La salinización de los suelos es un problema mundial y afecta casi un tercio del área dedicada a la agricultura. De los 230 millones de hectáreas regadas en el mundo, 45 millones de ha $(20 \%)$ han sido afectadas por la sal (Hoang et al., 2016). Las principales causas de la salinidad pueden incluir el cambio climático, el uso excesivo de agua subterránea para riego y drenaje (Cobos et al., 2020). Esta agua posee una elevada concentración de sales, que generalmente supera los límites de tolerancia de muchos cultivos a la sal, lo cual repercute en su producción (Ramírez-Suárez \& Hernández-Olivera, 2016).

La acumulación de sales solubles en el suelo afecta el crecimiento, la producción, el rendimiento y la sostenibilidad de muchos cultivos (Ramírez et al., 2017). La característica principal de los suelos salinos es la presencia de altas concentraciones de sales solubles, lo cual incrementa el potencial osmótico de la solución del suelo, causando estrés fisiológico, este tipo de suelos ofrece pocas opciones de crecimiento para las plantas, convirtiéndose en improductivos (Terrazas, 2018).

Los suelos con presencia de sales se clasifican de acuerdo a la conductividad eléctrica, como se muestra en la Tabla 1.

Tabla 1. Rangos de clasificación de suelos salinos.

\begin{tabular}{lc}
\hline Clasificación & CE $(\mathbf{d S} / \mathbf{m})$ \\
\hline Normales & 0 a 2 \\
Ligeramente salinos & 2 a 4 \\
Salinos & 4 a 8 \\
Fuertemente salinos & 8 a 16 \\
\hline
\end{tabular}

En Ecuador, la cuenca del río Guayas, que representa el $40.4 \%$ del área regable del país, tiene abundante agua, con un caudal de $8847 \mathrm{~m}^{3} / \mathrm{año}$; sin embargo, en los suelos de la cuenca hay acumulación de sales. Las condiciones de salinidad se deben a la intrusión salina del río Babahoyo, que ingresa por medio de los esteros y canales de riego, sumado a un drenaje deficiente (Pozo \& Sanfeliu, 2010). La productividad del arroz en este tipo de suelos es bastante baja, por lo que es importante determinar el nivel de sustentabilidad en suelos con y sin problemas de sales en los cantones de Yaguachi y Babahoyo, lo cual permitirá determinar diversas alternativas para reducir el efecto negativo causado por el estrés salino.

\section{Materiales y métodos}

Descripción del área de estudio

Cantón San Jacinto de Yaguachi (Figura 1), localidad afectada por sales, a una altura de $15 \mathrm{msnm}$, temperatura media anual $24.5-26{ }^{\circ} \mathrm{C}$ y precipitación media anual $750-1342 \mathrm{~mm}$.
Cantón Babahoyo (Figura 1), localidad libre de salinidad, a una altura de $8 \mathrm{msnm}$, temperatura media anual de $26.3{ }^{\circ} \mathrm{C}$, humedad relativa del $78.8 \%$, precipitación de $2688.8 \mathrm{~mm}$, evaporación de 1012.4 $\mathrm{mm}$, heliofanía de 830.4 horas y velocidad del viento de $0.5 \mathrm{~m} / \mathrm{seg}$. (INAMHI, 2019).

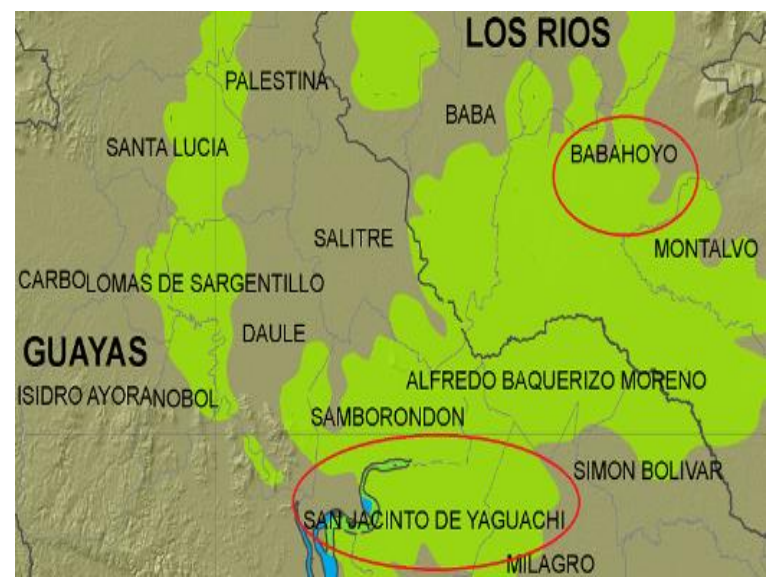

Fuente: Gobierno Autónomo Descentralizado Provincial de Los Ríos (GADPLR, 2019).

Figura 1. Ubicación de la zona de estudio.

\section{Metodología}

El estudio se desarrolló en dos fases.

FASE 1

Evaluación de aspectos físicos y químicos de suelo y agua para determinar los factores limitantes en la sostenibilidad del sector arrocero de Yaguachi y Babahoyo.

En la primera etapa del desarrollo de la investigación se aplicaron varios métodos y técnicas de trabajo de campo, para la evaluación de la salinidad del suelo y agua del sector arrocero en Yaguachi y en Babahoyo.

Se obtuvieron 10 muestras de suelo y 10 de agua utilizada para el riego en terrenos destinados al cultivo del arroz para los análisis físicos y químicos respectivos por localidad. Las coordenadas geográficas de las zonas de muestreo fueron determinadas con un equipo de geo posicionamiento (GPS).

En el caso de suelo se siguió el siguiente protocolo: limpieza de la superficie del sitio donde se tomaría la muestra de suelo, extracción de las submuestras con una pala haciendo un hoyo en forma de "V" hasta 20 $\mathrm{cm}$ de profundidad, toma de una tajada de suelo de 2 $3 \mathrm{~cm}$ de espesor con un cuchillo o machete, eliminación de los bordes y extracción de la parte central de la tajada (no mayor a $5 \mathrm{~cm}$ ). Se realizaron muestreos intensivos y sistemáticos en 10 fincas representativas de las dos localidades. En cada una de las fincas se marcaron 49 puntos de muestreo separados por una distancia de 100 $\mathrm{m}$. Las muestras se depositaron en un balde, para luego mezclarse de forma homogénea y considerar una 
muestra de $1 \mathrm{~kg}$ de suelo (muestra compuesta) para enviar al laboratorio (Córdova et al., 2020).

Para el muestro del agua se seleccionaron 10 áreas (Tabla 2), los sitios se seleccionaron utilizando el mapa del Cantón Yaguachi y el de Babahoyo. Las muestras se tomaron en la parte superficial de tres sitios diferentes, la cantidad fue de un litro en una botella de polietileno. Esta se llenó hasta al ras del envase, luego fue trasladada al laboratorio. Los puntos de muestreo se establecieron alrededor de los nacimientos de los canales de riego y terminaron en los campos en donde se aprovecha esta agua para irrigación.

FASE 2

Análisis de la sustentabilidad de dos sistemas de producción de arroz, uno en condiciones de salinidad del sector arrocero de Yaguachi y otro sin problemas de salinización en el Cantón Babahoyo.

Diagnósticico_del mananejo agrícola del_arroz. Para lo cual se aplicó una encuesta a dirigentes arroceros de las comunidades en estudio eligiendo a las personas de mayor edad con experiencia y conocimiento de la historia del lugar (Anzules, 2019).

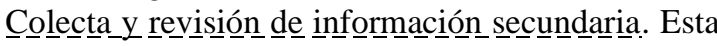
se realizó con representantes de instituciones de investigación del sector, como el Instituto Nacional de Investigaciones Agropecuarias (INIAP).

La experiencia de dirigentes arroceros de las comunidades en estudio, y representantes de instituciones de investigación del sector arrocero, permitió evaluar y ajustar los indicadores y subindicadores propuestos por Sarandón et al. (2006), los cuales fueron adaptados al cultivo de arroz en la zona de estudio.

Población y muestra

Según el SINAGAP (2014), el Cantón Yaguachi, en la Provincia del Guayas, posee 2235 Unidades de Producción Agropecuaria (UPA) de arroz, mientras que Babahoyo, en la Provincia de Los Ríos, mantiene actualmente 5133 UPA de este cultivo. Con el fin de llegar a una muestra representativa, se usó el método de proporciones empleando la fórmula propuesta por Scheaffer et al. (1987), citada por Parra \& Magaña (2019) (Fórmula 1). Acorde a ello, se realizaron 92 encuestas en el Cantón Yaguachi y 94 en el de Babahoyo, con un nivel de confianza del $90 \%$.

$$
\mathrm{n}=\frac{\mathrm{N} \times \sigma^{2}}{\frac{(\mathrm{N}-1) \times \mathrm{B}^{2}}{4+\sigma^{2}} \times 4}
$$

Fórmula 1. Método de proporciones para el cálculo del tamaño de muestra representativa.

Donde:

$\mathrm{N}$ : población o universo;

B: error relativo máximo esperado;

$\sigma$ : desviación del error \pm 3 .
Metodología para evaluar sustentabilidad

La metodología empleada fue "multicriterio", propuesta por Sarandón (2002), citada por Pinedo et al. (2020) que considera a la vez los lineamientos de Smyth \& Dumansky (1995). Se emplearon indicadores, subindicadores y variables cuantificables adaptadas al cultivo de arroz (Tablas 3, 4, 5 y 6), para analizar las dimensiones, económica, ecológica y sociocultural (Gómez et al., 2019). Las variables tuvieron valores de 0 a 4. Menos sustentable fue 0 (Sarandón et al., 2006; Caicedo et al., 2019; Caicedo et al., 2020). El Índice de sustentabilidad general (ISGen), fue calculado empleando las fórmulas propuestas por Sarandón \& Flores (2009) (Fórmulas 2, 3, 4 y 5).

FóRMULAS EMPLEADAS PARA EL CÁLCULO DE LOS INDICADORES DE SUSTENTABILIDAD

$\mathrm{IK}=\frac{2\left(\frac{\mathrm{A} 1+\mathrm{A} 2}{2}\right)+\mathrm{B}+\left(\frac{\mathrm{C} 1+\mathrm{C} 2+\mathrm{C} 3+\mathrm{C} 4+\mathrm{C} 5+\mathrm{C} 6}{6}\right)}{4}$

Fórmula 2. Indicador económico (IK) de Sarandón \& Flores (2009).

$$
\mathrm{IE}=\frac{\left(\frac{\mathrm{A} 1+\mathrm{A} 2}{2}\right)+B+\left(\frac{\mathrm{C} 1+\mathrm{C} 2}{2}\right)+\left(\frac{\mathrm{D} 1+\mathrm{D} 2+\mathrm{D} 3}{3}\right)}{4}
$$

Fórmula 3. Indicador Ecológico (IE) de Sarandón \& Flores (2009).

$$
\text { ISC }=\frac{2\left(\frac{\mathrm{A} 1+\mathrm{A} 2+\mathrm{A} 3+\mathrm{A} 4}{4}\right)+\mathrm{B}+\mathrm{C}+\mathrm{D}}{5}
$$

Fórmula 4. Indicador Sociocultural (ISC) de Sarandón \& Flores (2009).

$$
\mathrm{ISGen}=\frac{\mathrm{IK}+\mathrm{IE}+\mathrm{ISC}}{3}
$$

Fórmula 5. Índice de sustentabilidad general (ISGen) de Sarandón \& Flores (2009).

\section{Resultados}

Fase 1. Aspectos físicos y químicos de suelo y agua

SUELO

TEXTURA

Para las dos localidades, con 20 sitios de muestreo, la clase textural predominante fue arcilla $(70 \%$ de los casos) a la profundidad de 0 a $20 \mathrm{~cm}$ (Tabla 7). Los suelos de estas localidades son clasificados como vertisoles arcillosos Gley, con más de $35 \%$ de arcilla, muy plástica y pegajosa, con más de $50 \%$ de la fracción de arcilla expandible (2:1), con severo agrietamiento e hinchamiento, mal drenados, saturados con agua, estado que en algunos casos se mantiene por más de 60 días; además, presenta moteados con cromas $<2$ en los primeros $60 \mathrm{~cm}$ y debajo de los horizontes $\mathrm{AB}$ (Mejía et al., 1997). 


\section{MACRO Y MICROELEMENTOS}

Respecto a los macro elementos: fósforo (P), potasio $(\mathrm{K})$, calcio $(\mathrm{Ca})$, magnesio $(\mathrm{Mg})$ y azufre $(\mathrm{S})$. Se puede apreciar mayores valores en la zona de Yaguachi. Probablemente esto se debe a la acumulación de materiales finos arrastrados por las lluvias desde las zonas altas, y esos materiales se encuentran asociados a bases cambiables ( $\mathrm{Ca}, \mathrm{K}, \mathrm{Mg}$ ).

Con respecto al contenido de micro elementos en las zonas evaluadas, se determinó una tendencia contraria a la señalada para los macro elementos, esto es, en el sector de Babahoyo los contenidos de cobre $(\mathrm{Cu})$, hierro $(\mathrm{Fe})$, manganeso $(\mathrm{Mn})$, Zinc $(\mathrm{Zn})$ y boro (B) fueron mayores que en el de Yaguachi. Estos resultados están relacionados con la movilización de los microelementos metálicos $(\mathrm{Cu}, \mathrm{Mn}, \mathrm{Zn}, \mathrm{Fe}$,) los cuales se movilizan preferentemente a través del sistema orgánico en los suelos, mientras que los macro elementos $(\mathrm{K}, \mathrm{Ca}, \mathrm{Mg})$ lo hacen a través del sistema inorgánico (Tabla 8).

\section{PH Y CONDUCTIVIDAD ELÉCTRICA}

En la zona de Yaguachi según los análisis de $\mathrm{pH}$, se puede apreciar que estos varían de 5.32 a 7.51. Con respecto a la conductividad eléctrica (CE en $\mathrm{dS} / \mathrm{m}$ ) se aprecia un rango de 0.3 a 9.4, siendo estos los valores más altos de CE. Los suelos de esta localidad son clasificados como salinos y fuertemente salinos causando efectos negativos en el comportamiento de los cultivos de arroz que se realizan en estos sitios. Estos suelos se caracterizan por un $\mathrm{pH}$ de carácter alcalino, con contenido sódico elevado (psi > 30\%; psi: libras por pulgada cuadrada) (Briones et al., 2010).

Por otro lado, los suelos del sector de Babahoyo, tienen niveles de conductividad eléctrica que en ningún caso supera los $4 \mathrm{dS} / \mathrm{m}$ y son clasificados como ligeramente salinos y normales (Tabla 9).

\section{COMPACTACIÓN}

Los valores de compactación en Yaguachi, entre los 0 y $30 \mathrm{~cm}$ de profundidad del suelo presentaron un rango de 180 a 300 psi; es decir, los suelos en general presentan un grado de compactación elevado, el cual tiene un efecto negativo en el desarrollo de las raíces. El riego, la mecanización, la aplicación de fertilizantes y otras actividades mal balanceadas, son la causa fundamental de la degradación física, manifestándose por compactación, erosión y mal drenaje del suelo (Martínez et al., 2019).

En el caso de Babahoyo, los valores variaron de 120 a 180 psi: estos valores indican que los suelos tienen en general menor grado de compactación, lo cual permite una mayor infiltración y exploración de raíces (Tabla $10)$.

\section{AGUA}

Para Babahoyo el pH (Tabla 11) varió de 6.6 a 7.9 y la CE de 0.2 a $0.9 \mathrm{dS} / \mathrm{m}$. El agua de riego de esta zona se clasifica como de bajo riesgo de salinidad, con poca probabilidad de alcanzar niveles peligrosos de sodio intercambiable, por lo que puede utilizarse para el riego siempre y con cierto grado de lavado. Las plantas moderadamente tolerantes a las sales pueden producir adecuadamente sin prácticas de control de salinidad.

En Yaguachi el pH del agua varió de 6.4 a 7.16 y la $\mathrm{CE}$ de 2.09 a $3.52 \mathrm{dS} / \mathrm{m}$. Para el laboratorio de Salinidad de Riverside (USA), la mayoría de aguas para riego del cantón están clasificadas como C2, aguas de salinidad media que pueden usarse para riego bajo la condición de que exista por lo menos un lavado moderado de los suelos. Un porcentaje bajo está clasificado como C3 de agua de salinidad alta que puede utilizarse para el riego de suelos con buen drenaje, empleando volúmenes de agua en exceso para lavar el suelo y utilizando cultivos muy tolerantes a la salinidad (Tabla 12).

En general, de acuerdo a la clasificación de la FAO (1997), el pH de las aguas muestreadas que llegan a las parcelas se encuentra dentro de la amplitud normal (6.6 - 8.2) de neutro a moderadamente alcalino por lo que no es un factor limitante para el normal desarrollo de las plantas.

Fase 2. Análisis de la sustentabilidad

Las Tablas 13, 14 y 15 muestran el resumen de los resultados de la caracterización de los agroecosistemas, en sus dimensiones económica productiva, ambiental y sociocultural, respectivamente.

\section{DIMENSIÓN ECONÓMICA}

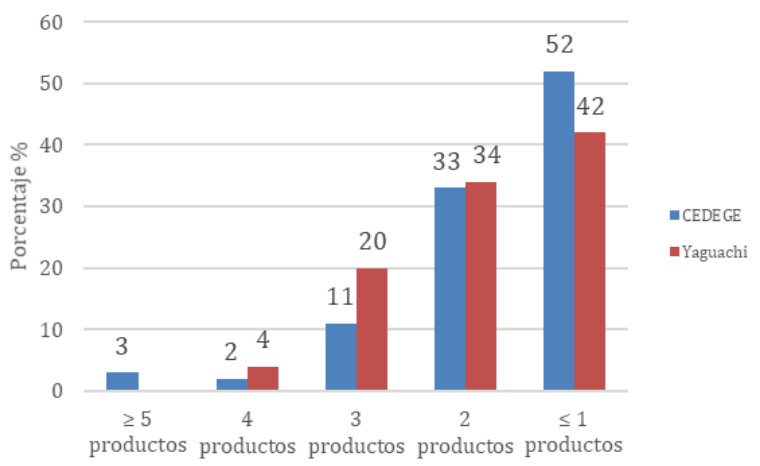

Figura 2. Diversificación de la producción.

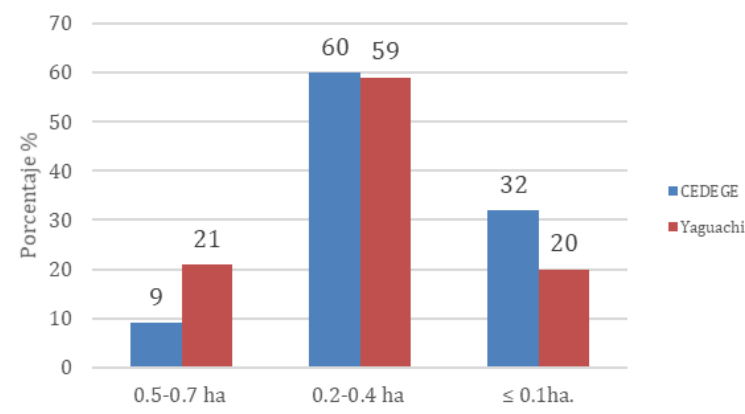

Figura 3. Superficie de producción destinada al autoconsumo. 


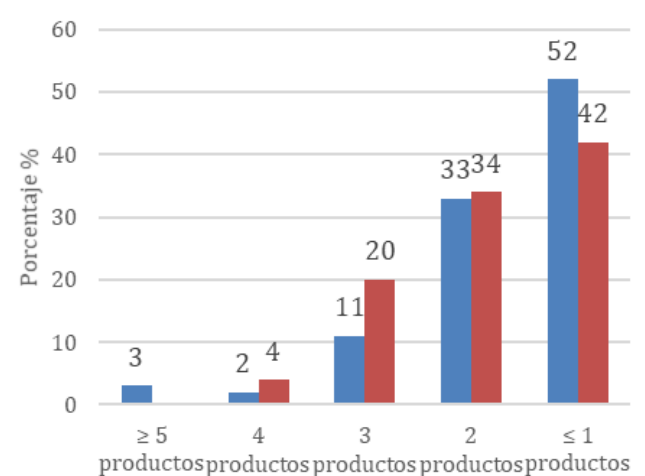

Figura 4. Diversificación para la venta.

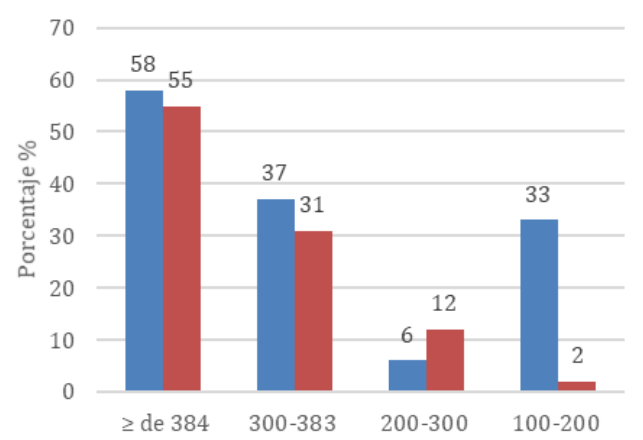

Figura 5. Ingreso mensual.

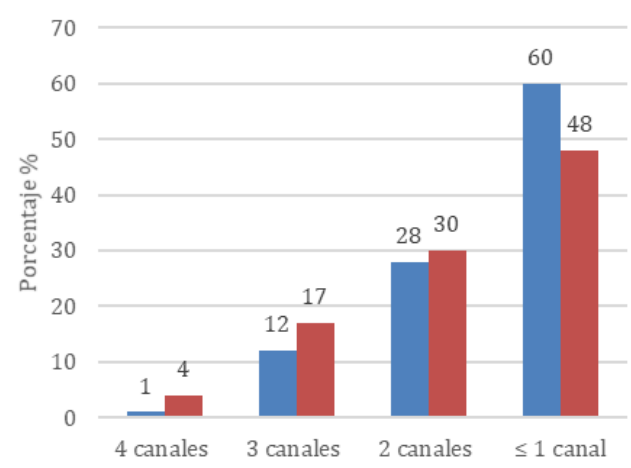

Figura 6. Vías de comercialización.

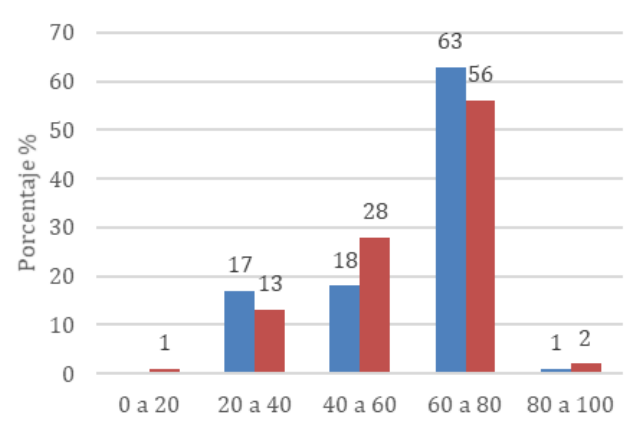

(\%)

Figura 7. Dependencia de insumos externos.

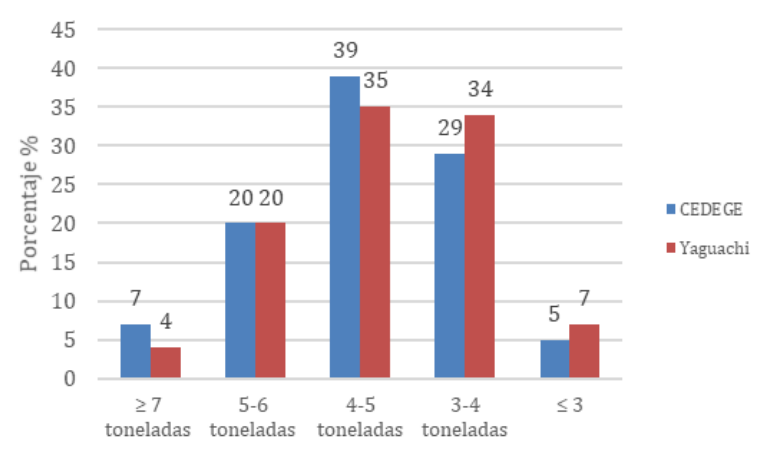

Figura 8. Productividad / área.

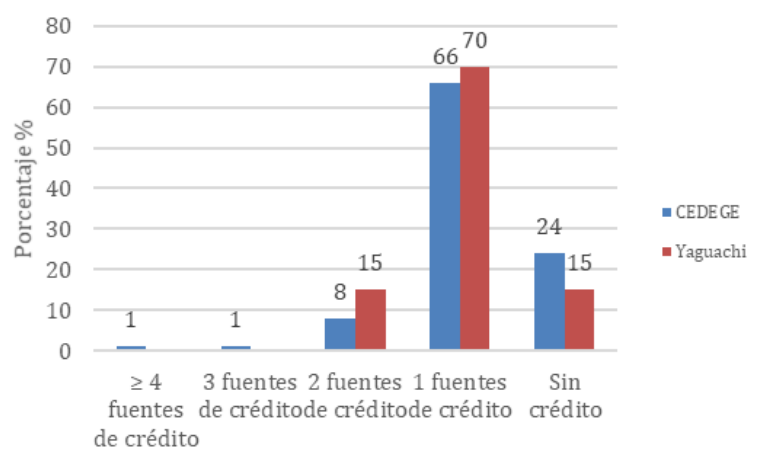

Figura 9. Fuentes de crédito.

\section{DIMENSIÓN ECOLÓGICA}

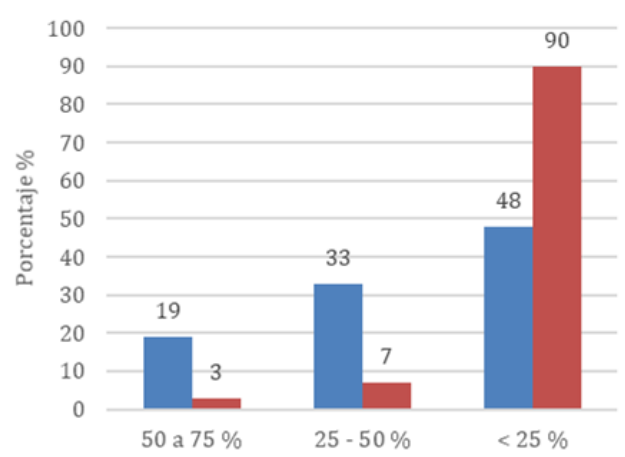

Figura 10. Manejo de la cobertura vegetal.
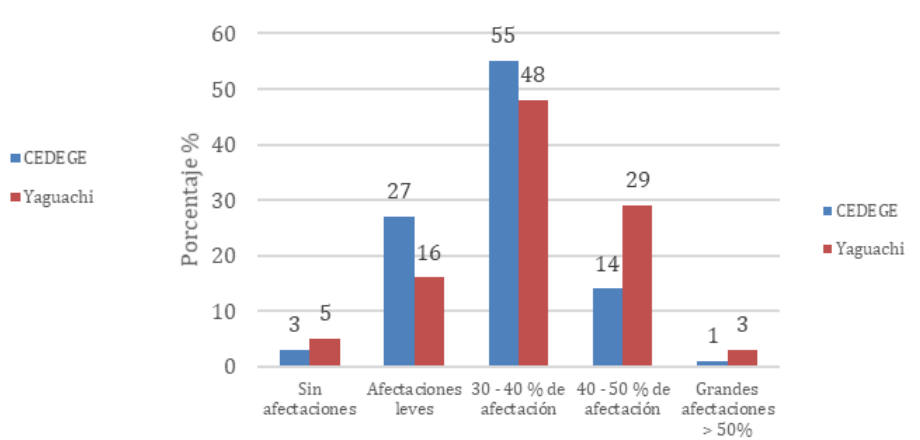

Figura 11. Rotación de cultivos. 


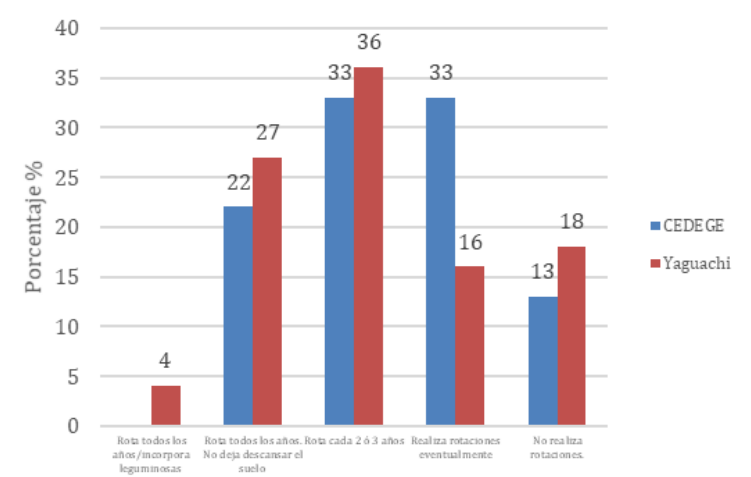

Figura 12. Incidencia de plagas.

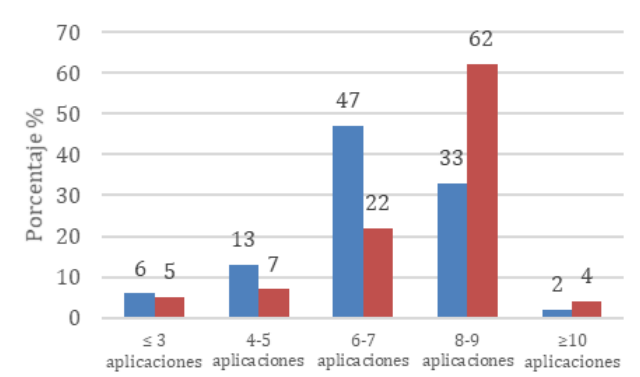

Figura 13. Aplicaciones Yaguachi.

\section{DIMENSIÓN SOCIOCULTURAL}

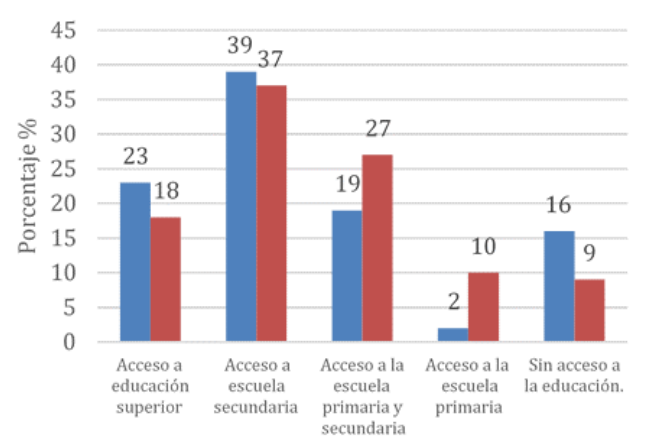

Figura 14. Acceso a la educación.

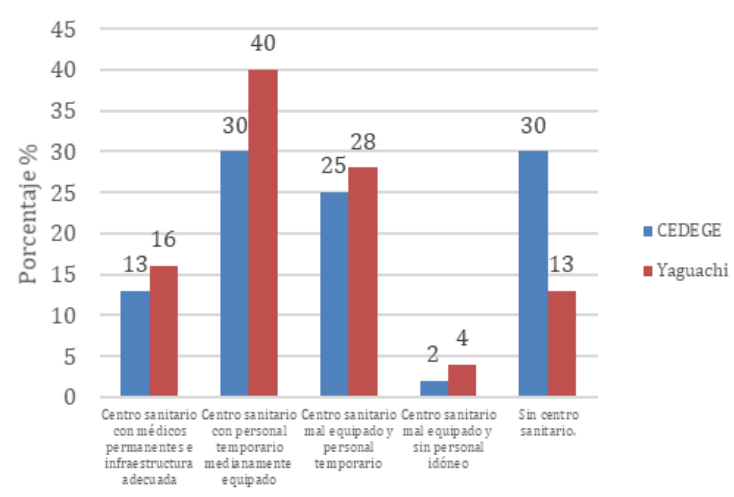

Figura 15. Acceso a salud.

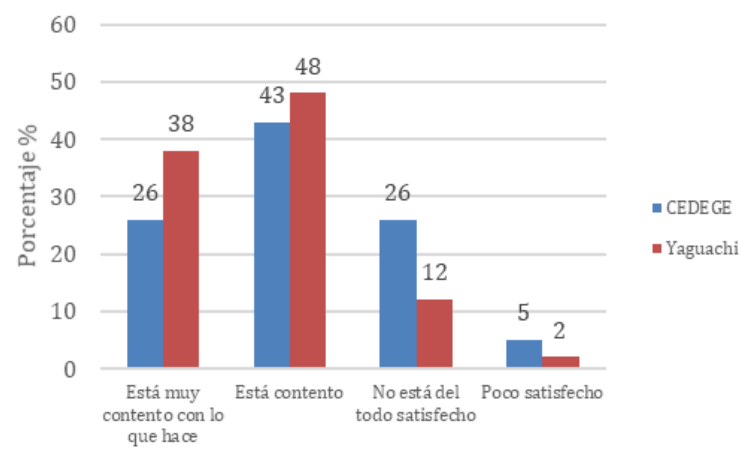

Figura 16. Aceptabilidad del sistema de producción.

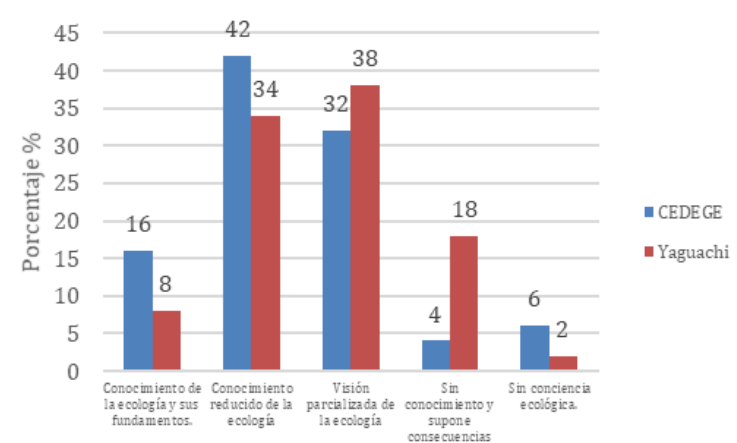

Figura.17. Conocimiento y conciencia ecológica.

Tipificación de fincas arroceras en el Cantón Babahoyo

La tipificación se realizó empleando 39 variables con un coeficiente de variación superior a $60 \%$.

Al aplicar el método Ward y la distancia euclidiana al cuadrado, comparando con lo expresado por Martínez (2013), se formaron 3 grupos de sistemas, como se muestra en la Figura 18, de los cuales el primero corresponde al segmento de productores del sistema de producción desarrollado (SPD) con 14\%, el conglomerado 2 corresponde a sistemas de producción medianamente desarrollado (SPMD) con 13\% y el tercero agrupa la mayor parte de productores que conforma el sistema de producción básica (SPB) con $73 \%$.

Tipificación de fincas arroceras en el Cantón Yaguachi

Siguiendo el procedimiento descrito anteriormente, se formaron 3 grupos para este cantón, lo cual se muestra en la Figura 19. El primero corresponde al sistema de producción desarrollado (SPD) con 13\%, el conglomerado 2 corresponde a sistema de producción medianamente desarrollado (SPMD) con $58 \%$ y el tercero al sistema de producción básica (SPB) con $29 \%$. Evaluación de la sustentabilidad General

Las Figuras 20, 21, 22, 23, 24 y 25 presentes en este apartado, muestran el resultado del análisis de la información obtenida en la investigación. Utilizando los indicadores y sub indicadores ya establecidos, se procedió a valorar según la escala propuesta a cada uno de ellos, empleando las Fórmulas 2, 3, 4 y 5 respectivamente para las dimensiones sociales, ecológicas y económicas, obteniendo los índices de 
sustentabilidad, graficados en esquemas tipo ameba donde se establecen los puntos críticos de sustentabilidad inferiores o iguales a dos y los indicadores con sustentabilidad alta, igual o superior a tres.

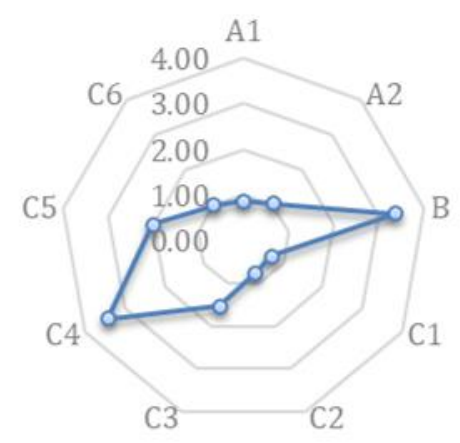

\begin{tabular}{|l|lll|l|l|l|l|l|}
\hline A1 & A2 & B & C1 & C2 & C3 & C4 & C5 & C6 \\
\hline
\end{tabular}

\begin{tabular}{|l|lll|l|l|l|l|l|l|}
\hline 0.86 & 1.01 & 3.38 & 0.74 & 0.77 & 1.53 & 3.34 & 1.79 & 1.00 \\
\hline
\end{tabular}

Figura 20. Indicador Económico Yaguachi: 1.7.

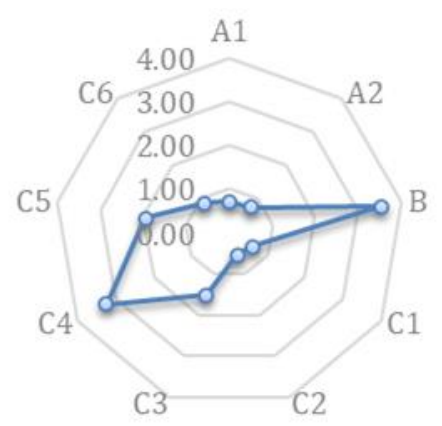

\begin{tabular}{c|c|c|c|c|c|c|cc|}
\hline A1 & A2 & B & C1 & C2 & C3 & C4 & C5 & C6 \\
\hline 0.71 & 0.77 & 3.52 & 0.62 & 0.54 & 1.52 & 3.25 & 1.95 & 0.88 \\
\hline
\end{tabular}

Figura 21. Indicador Económico Babahoyo: 1.6.

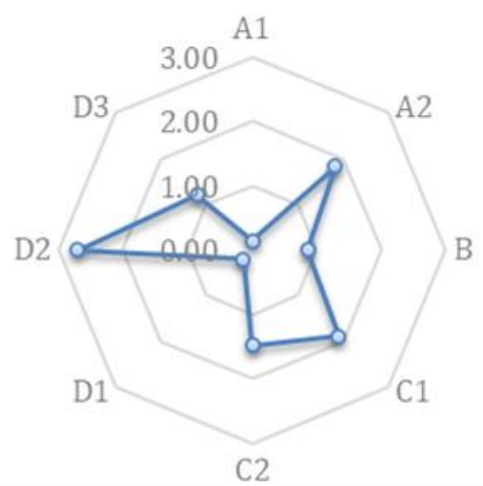

\begin{tabular}{c|c|c|c|c|c|c|c|}
\hline A1 & A2 & B & C1 & C2 & D1 & D2 & D3 \\
\hline 0.13 & 1.83 & 0.86 & 1.90 & 1.48 & 0.20 & 2.70 & 1.20 \\
\hline
\end{tabular}

Figura 22. Indicador Ecológico Yaguachi: 1.2.

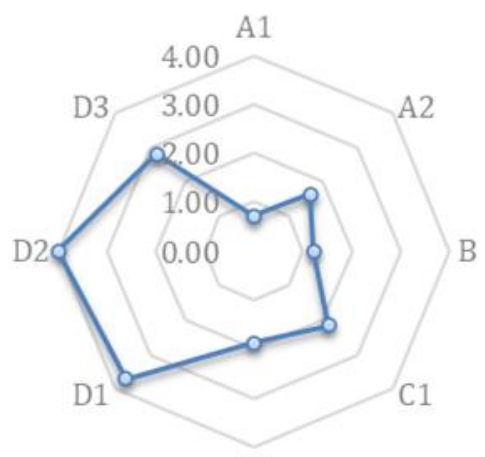

$\mathrm{C} 2$

\begin{tabular}{|c|c|c|c|c|c|c|c|}
\hline A1 & A2 & B & C1 & C2 & D1 & D2 & D3 \\
\hline 0.71 & 1.64 & 1.22 & 2.16 & 1.88 & 3.70 & 4.00 & 2.80 \\
\hline
\end{tabular}

Figura 23. Indicador Ecológico Babahoyo: 2.0.

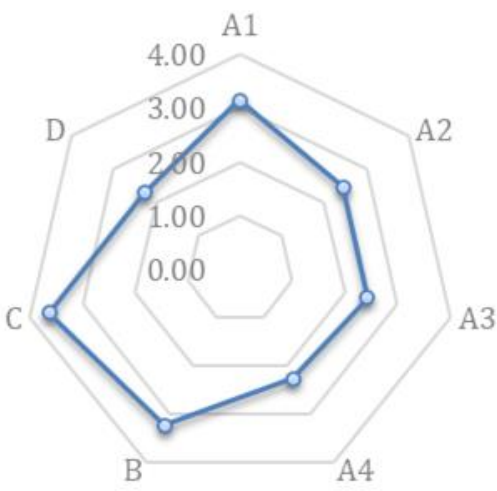

\begin{tabular}{|c|cc|c|c|c|c|}
\hline A1 & A2 & A3 & A4 & B & C & D \\
\hline 3.14 & 2.45 & 2.42 & 2.27 & 3.23 & 3.64 & 2.27 \\
\hline
\end{tabular}

Figura 24. Indicador Sociocultural Yaguachi: 2.9.

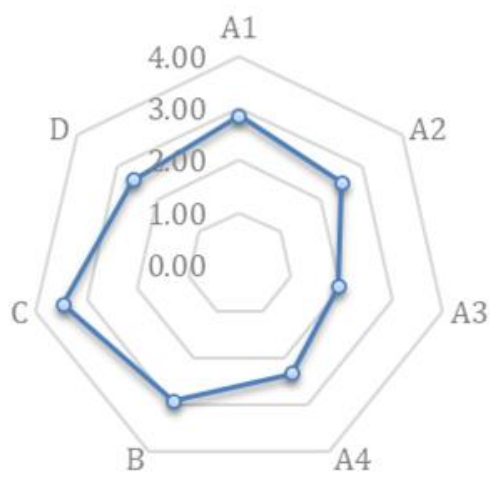

\begin{tabular}{|c|c|c|c|c|c|c|}
\hline A1 & A2 & A3 & A4 & B & C & D \\
\hline 2.86 & 2.51 & 1.95 & 2.32 & 2.90 & 3.47 & 2.60 \\
\hline
\end{tabular}

Figura 25 Indicador Sociocultural Babahoyo: 2.8.

En el caso de los sistemas arroceros evaluados de Yaguachi y Babahoyo, los dos sistemas alcanzan un ISGen de 1.93 (Figura 26) y 2.12 (Figura 27), respectivamente. Sin embargo, no se consideran 
sustentables, debido a que en los dos sistemas existen dimensiones con valores menores a dos.

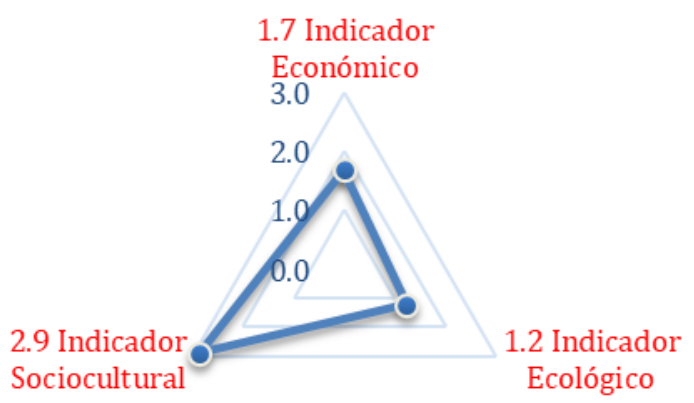

Figura 26. Indicadores económico, ecológico y sociocultural para la zona de Yaguachi $($ ISGen $=1.93$ ).

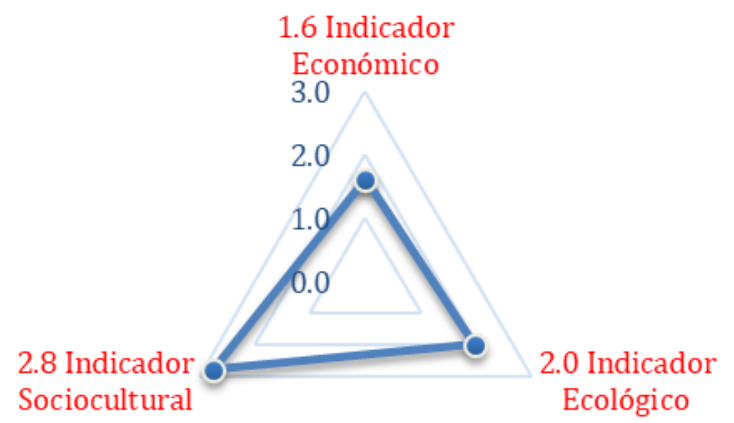

Figura 27. Indicadores económico, ecológico y sociocultural para la zona de Babahoyo (ISGen $=2.12$ ).

\section{Conclusiones}

La práctica común en el cultivo de arroz en los Cantones de Yaguachi y Babahoyo es la siembra directa al voleo y la aplicación del sistema de riego por inundación.

Yaguachi tiene problemas de salinización, sus suelos son fuertemente salinos, en el orden del $70 \%$ y $30 \%$, respectivamente. Este fenómeno se refleja en el crecimiento de los cultivos en parches, disminuyendo la productividad del área con respecto al Cantón Babahoyo y afectando la economía de las comunidades aledañas.

Entre las principales causas de la salinidad de los suelos, encontradas en el sector de arroceros de Yaguachi, está la utilización de aguas salinas provenientes del río Yaguachi para el riego del cultivo, lo que ocasiona la acumulación de sales en la superficie de dichos suelos. El pH del agua varió de 6.4 a 7.16 y la $\mathrm{CE}$ de 2.09 a $3.52 \mathrm{dS} / \mathrm{m}$, clasificada como agua con problemas de sales.

Sobre el nivel de sustentabilidad del cultivo de arroz, el Índice de Sustentabilidad General para Yaguachi fue de 1.93 y para Babahoyo, de 2.12; valores determinados mediante el análisis de las dimensiones económicas, ecológicas y socioculturales, mostrando diferencias entre la zona salinizada y la no salinizada.

En el sector de arroceros de los cantones de Yaguachi y Babahoyo se determinaron tres sistemas de producción: sistema de producción desarrollado (SPD), sistema de producción medianamente desarrollado (SPMD) y sistema de producción básica (SPB).

El uso de enmiendas orgánicas puede ser útil en el manejo de suelos irrigados con aguas salinas, ya que poseen efectos positivos sobre el suelo y por sus características químicas poseen un buen potencial para la remediación de la sodicidad.

\section{Literatura citada}

Anzules V. 2019. Sustentabilidad de sistemas de producción de cacao (Theobroma cacao L.) en Santo Domingo de los Tsáchilas, Ecuador. Tesis de Doctorado. Universidad Nacional Agraria La Molina.

http://repositorio.lamolina.edu.pe/bitstream/handle/UNA LM/4110/anzules-toala-vicente-

paul.pdf? sequence $=1 \&$ isAllowed $=\mathrm{y}$.

Briones C., Proaño J. \& Medina K. 2010. Determinación de las aptitudes físicas y químicas en distintas unidades taxonómicas de suelos en la hacienda campo Alegre (Quilichao), Parroquia Taura, Cantón Naranjal. Provincia del Guayas. XII Congreso Ecuatoriano de la Ciencia del Suelo. Sociedad Ecuatoriana de la Ciencia del Suelo. http://www.secsuelo.org/wpcontent/uploads/2015/06/11.-Jaime-Proano.-Suelos.Agraria.pdf.

Caicedo-Camposano O., Díaz-Romero O., Cadena-Piedrahita D. \& Galarza-Centeno G. 2019. Diseño de un sistema de producción de arroz sostenible en Babahoyo, Provincia de Los Ríos, Ecuador. Killkana Técnica, 3(1): 19-24. https://doi.org/10.26871/killkana_tecnica.v3i1.472.

Caicedo-Camposano O., Soplín-Villacorta H., BalmasedaEspinosa C., Cadena-Piedrahita L. \& Leyva-Vázquez M. 2020. Sustentabilidad de sistemas de producción de banano (Musa paradisiaca AAA) en Babahoyo, Ecuador. Revista Investigación Operacional, 41(3): 379-388. https://rev-invope.pantheonsorbonne.fr/sites/default/files/inlinefiles/41320-07.pdf.

Cobos F., Gómez L., Reyes W. \& Hasang E. 2020. Evaluación de la tolerancia a la salinidad en poblaciones segregantes F5 de arroz (Oryza sativa L.). Journal of Science and Research, 5(5: num. especial del I CININGEC): 1-23. https://revistas.utb.edu.ec/index.php/sr/article/view/995.

Cobos F., Hasang E., Lombeida E. \& Medina R. 2021. Caracterización de fincas arroceras en sistemas de producción bajo riego, en el Cantón Daule. Journal of Science and Research, 5(5: num. especial del I CININGEC): 156-168. https://revistas.utb.edu.ec/index.php/sr/article/view/1004 
Córdova C., Magna C., Barrera J. \& Zagal E. 2020. Dependencia espacial del potencial de nitrógeno disponible del suelo en dos sitios contrastantes. Chilean journal of agricultural \& animal sciences, 36(1): 14-25. http://agro-ciencia.cl/web/wp-

content/uploads/2020/03/AC-1940.-Potencial-denitrogeno-28.03.2020.pdf.

FAO. 1997. Management of Agricultural Drainage Water Quality. Water Report 13, Food and Agriculture Organization, http://www.fao.org/3/w7224e/w7224e00.htm.

FAO. 2012. Guía para la descripción de suelos. FAO. Rome. http://www.fao.org/3/a0541s/a0541s.pdf.

FAOSTAT. 2018. Food and Agriculture Organization statistical database. Consultado el 30 mayo del 2018 de: http://faostat.fao.org/default.aspx.

GADPLR (Gobierno Autónomo Descentralizado Provincial de Los Ríos). 2019. Plan de desarrollo y ordenamiento territorial 2015 - 2019, Ecuador. http://app.sni.gob.ec/snilink/sni/PORTAL_SNI/data_sigad_plus/sigadplusdocum entofinal/1260000140001_PDyOT\%20FINAL\%20GAD PLR\%20-2015\%20final_15-05-2016_08-47-09.pdf.

Gómez J., Cobos F. \& Hasang E. 2019. Sostenibilidad de los sistemas de producción de ganadería extensiva. Journal of Science and Research, 4(5: CIEIS2019): 180-195. https://revistas.utb.edu.ec/index.php/sr/article/view/798.

Hoang T., Tran T., Nguyen T., Williams B., Wurm P., Bellairs S. \& Mundree S. 2016. Improvement of salinity stress tolerance in rice: Challenges and opportunities. Agronomy, 6(4): 54. DOI: 10.3390/agronomy6040054.

INAMHI (National Institute of Meteorology and Hydrology). 2019. Agrometeorology Station of the Faculty of Agricultural Sciences of the Technical University of Babahoyo, Los Ríos, Ecuador. Consultado el 10 de Abril de 2019 de:

http://www.serviciometeorologico.gob.ec/boletinesmeteorologicos/.

INEC (Instituto Nacional de Estadística y Censos). 2020. Encuesta de Superficie y Producción Agropecuaria Continua (ESPAC) 2019 https://www.ecuadorencifras.gob.ec/documentos/webinec/Estadisticas_agropecuarias/espac/espac2019/Presentacion\%20de\%20los\%20principales\%20res ultados\%20ESPAC\%202019.pdf.

Martínez M., Osuna E. \& Espinosa M. 2019. Impacto acumulado de la agricultura de conservación en propiedades del suelo y rendimiento de maíz. Revista mexicana de ciencias agrícolas, 10(4): 765-778. https://doi.org/10.29312/remexca.v10i4.1640.

Martínez L. 2013. La Agricultura Familiar en el Ecuador. Informe del Proyecto Análisis de la Pobreza y de la Desigualdad en América Latina Rural. SERIE DOCUMENTOS DE TRABAJO. Documento $\mathrm{N}^{\circ} 147$. Grupo de Trabajo: Desarrollo con Cohesión Territorial. Rimisp (Centro Latiniamericano para el Desarrollo rural). Quito. Ecuador. http://rimisp.org/wpcontent/files_mf/1434745799147AgriculturaFamiliarEc uadorMartinez_editado.pdf.
Mejía V. L., Instituto Geográfico Militar (Ecuador) \& Fundación Forestal Juan Manuel Durini. 1997. Suelos del Ecuador: Reconocimiento general en base a su capacidadfertilidad y mapa general de clasificación por capacidadfertilidad: una interpretación básica sobre las características de los suelos del Ecuador. Instituto Geográfico Militar. Quito, Ecuador.

Parra R. \& Magaña M. 2019. Características técnicoeconómicas de los sistemas de producción bovina basados en razas criollas introducidas en México. Ecosistemas y recursos agropecuarios, 6(18): 535-547. https://doi.org/10.19136/era.a6n18.2160.

https://era.ujat.mx/index.php/rera/article/view/2160.

Pinedo R., Gómez L. \& Julca A. 2020. Sostenibilidad ambiental de la producción de quinua (Chenopodium quinoa Willd.) en los valles interandinos del Perú. Ciencia y Tecnología Agropecuaria, 21(3): art 1309. https://doi.org/10.21930/rcta.vol21_num3_art:1309.

Pozo W. \& Sanfeliu T. 2010. Variabilidad Espacial Temporal de la Salinidad del Suelo en los Humedales de Arroz en la Cuenca Baja del Guayas, Sudamérica. Revista Tecnológica ESPOL, 23(1): 73-79.

http://www.rte.espol.edu.ec/index.php/tecnologica/articl e/view/38.

Ramírez M., Urdaneta A. \& Pérez E. 2017. Germinación del guayabo tipo "criolla roja" bajo condiciones de salinidad por cloruro de sodio. BIOAGRO, 29(1): 65-72. http://www.ucla.edu.ve/bioagro/Rev29(1)/8.\%20ms\%20 1605.pdf. http://ve.scielo.org/scielo.php?pid=S131633612017000100008\&script=sci_abstract.

Ramírez-Suárez W. \& Hernández-Olivera L. 2016. Tolerancia a la salinidad en especies cespitosas. Pastos y Forrajes, 39(4): 235-245.

http://scielo.sld.cu/scielo.php?script=sci_arttext\&pid=S0 864-03942016000400001.

Sarandón S. \& Flores C. 2009. Evaluación de la sustentabilidad en agroecosistemas: una propuesta metodológica. Revista Agroecología, 4: 19-28. Madrid, España.

https://revistas.um.es/agroecologia/article/view/117131.

Sarandón S., Zuluaga M., Cieza R., Gómez C., Janjetic L. \& Negrete E. 2006. Evaluación de la sustentabilidad de sistemas agrícolas de fincas en Misiones, Argentina, mediante el uso de indicadores. Agroecología, 1: 19-28. https://revistas.um.es/agroecologia/article/view/14.

SINAGAP (Sistema de Información Nacional de Agricultura y Ganadería). 2014. III Censo Nacional Agropecuario: Referencias del levantamiento censal. Consultado el 5 de julio de 2017 de: http://sinagap.agricultura.gob.ec/censonacional-agropecuario.

Smyth A. \& Dumansky G. 1995. A framework for evaluating sustainable land management. Canadian Journal of Soil Science., 75(4): 401-406. DOI: 10.4141/cjss95-059.

Terrazas J.M. 2018. Efecto de tres niveles de salinidad en el crecimiento del pasto agropiro variedad Alkar (Thinopyrum ponticum) mediante reproducción sexual y vegetativa. Apthapi, 4(3): 1295-1311. http://ojs.agro.umsa.bo/index.php/ATP/article/view/261. 
Enero - Julio 2021

\begin{tabular}{|c|c|c|c|c|c|}
\hline CÓDIGO & RECINTO & PROPIETARIO & CANTÓN & PROVINCIA & $\begin{array}{l}\text { COORDENADAS } \\
\text { GEOGRÁFICAS }\end{array}$ \\
\hline P1 & Providencia & Huly Ruiz & Yaguachi & Guayas & $\begin{array}{r}2^{\circ} 4^{\prime} 21.73^{\prime \prime} \mathrm{S} \\
79^{\circ} 39^{\prime} 15.52^{\prime \prime} \mathrm{O}\end{array}$ \\
\hline $\mathrm{P} 2$ & Hcda. Palo largo & Jairo de Jesús & Yaguachi & Guayas & $\begin{array}{r}2^{\circ} 0{ }^{\prime} 27.662 \text { '’ } \mathrm{S} \\
79^{\circ} 43^{\prime} 21.48^{\prime \prime} \mathrm{O}\end{array}$ \\
\hline P3 & Coop. San Jacinto & Víctor Moncada & Yaguachi & Guayas & $\begin{array}{r}2^{\circ} 6^{\prime} 45.62, ' \mathrm{~S} \\
79^{\circ} 45^{\prime} 20.13 \text { ' } \mathrm{O}\end{array}$ \\
\hline $\mathrm{P} 4$ & El Chobo & Sr. Noe Zapata & Yaguachi & Guayas & $\begin{array}{r}2^{\circ} 8{ }^{\prime} 40.49 \text { '’S } \\
79^{\circ} 38^{\prime} 0.13 \text { '” }\end{array}$ \\
\hline P5 & $\mathrm{Km} 5$ & Jorge Quiroz & Yaguachi & Guayas & $\begin{array}{r}2^{\circ} 6{ }^{\prime} 19.68^{\prime \prime} \mathrm{S} \\
79^{\circ} 40^{\prime} 59.34, ' \mathrm{O}\end{array}$ \\
\hline P6 & 4 hermanos & César Murillo & Yaguachi & Guayas & $\begin{array}{r}2^{\circ} 4{ }^{\prime} 38.40^{\prime \prime} \mathrm{S} \\
79^{\circ} 42^{\prime} 45.63^{\prime \prime} \mathrm{O}\end{array}$ \\
\hline P7 & Rcto. Vuelta Larga & Wilson Quezada & Yaguachi & Guayas & $\begin{array}{r}2^{\circ} 9^{\prime} 3.95 \text { ', } \mathrm{S} \\
79^{\circ} 40^{\prime} 29.68^{\prime \prime} \mathrm{O}\end{array}$ \\
\hline P8 & Rcto. La Lola & Pedro Contreras & Yaguachi & Guayas & $\begin{array}{r}2^{\circ} 3{ }^{\prime 2} 27.99 \text { '’ } \\
79^{\circ} 43^{\prime} 5.41 \text { ' } \mathrm{O}\end{array}$ \\
\hline P9 & Rcto. Las Mercedes & Fernando Espinoza & Yaguachi & Guayas & $\begin{array}{r}2^{\circ} 0{ }^{\prime} 5.428, ' \mathrm{~S} \\
79^{\circ} 377^{\prime} 51.48^{\prime \prime} \mathrm{O}\end{array}$ \\
\hline P10 & Caimito & Julio Arreaga & Yaguachi & Guayas & $\begin{array}{r}2^{\circ} 0{ }^{\prime} 25.19^{\prime \prime} \mathrm{S} \\
79^{\circ} 38^{\prime} 45.86^{\prime \prime} \mathrm{O}\end{array}$ \\
\hline P11 & Sabaneta & Jorge Quiroz & Babahoyo & Los Ríos & $\begin{array}{r}1^{\circ} 50^{\prime} 43.38^{\prime \prime} \mathrm{S} \\
79^{\circ} 26^{\prime} 49.00^{\prime \prime} \mathrm{O}\end{array}$ \\
\hline $\mathrm{P} 12$ & Sabaneta & Sr. José Banderas & Babahoyo & Los Ríos & $\begin{array}{r}1^{\circ} 51 ' 39.58^{\prime \prime} \mathrm{S} \\
79^{\circ} 27^{\prime} 37.20^{\prime \prime} \mathrm{O}\end{array}$ \\
\hline P13 & Playas & Félix Montero & Babahoyo & Los Ríos & $\begin{array}{r}1^{\circ} 51^{\prime} 56.26^{\prime \prime} \mathrm{S} \\
79^{\circ} 25^{\prime} 43.49 \text { '’ }\end{array}$ \\
\hline P14 & Playas & Sr. José Valero & Babahoyo & Los Ríos & $\begin{array}{r}1^{\circ} 51 ' 59.97 \text { '’ } \\
79^{\circ} 26^{\prime} 28.60^{\prime \prime} \mathrm{O}\end{array}$ \\
\hline P15 & Cedege & David Mendoza & Babahoyo & Los Ríos & $\begin{array}{r}1^{\circ} 53 ' 31.38^{\prime \prime} \mathrm{S} \\
79^{\circ} 27^{\prime} 27.31^{\prime \prime} \mathrm{O}\end{array}$ \\
\hline P16 & La Corona & Raúl Ramírez & Babahoyo & Los Ríos & $\begin{array}{l}1^{\circ} 53 ' 19.03, ' \mathrm{~S} \\
79^{\circ} 28^{\prime} 3.16, ' \mathrm{O}\end{array}$ \\
\hline P17 & San Pablo & Sr. Juan Andrade & Babahoyo & Los Ríos & $\begin{array}{r}1^{\circ} 52^{\prime} 48.76^{\prime \prime} \mathrm{S} \\
79^{\circ} 25^{\prime} 51.53^{\prime \prime} \mathrm{O}\end{array}$ \\
\hline P18 & El Palmar & Sr. Marco Calero & Babahoyo & Los Ríos & $\begin{array}{l}1^{\circ} 52^{\prime} 13.25^{\prime \prime} \mathrm{S} \\
79^{\circ} 27^{\prime} 4.45^{\prime \prime} \mathrm{O}\end{array}$ \\
\hline P19 & El Palmar & José Velasco & Babahoyo & Los Ríos & $\begin{array}{r}1^{\circ} 53^{\prime} 0.50 \text { '”S } \\
79^{\circ} 26^{\prime} 40.96^{\prime \prime} \mathrm{O}\end{array}$ \\
\hline $\mathrm{P} 20$ & El Palmar & Duval Cabrera & Babahoyo & Los Ríos & $\begin{array}{l}1^{\circ} 52^{\prime} 56.79^{\prime \prime} \mathrm{S} \\
79^{\circ} 28^{\prime} 5.63^{\prime \prime} \mathrm{O}\end{array}$ \\
\hline
\end{tabular}


Tabla 3. Subindicadores y variables para evaluar el Indicador Económico.

\begin{tabular}{|c|c|c|c|c|c|c|c|c|c|}
\hline \multirow{4}{*}{ 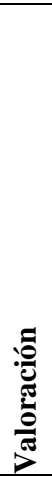 } & \multicolumn{2}{|c|}{$\begin{array}{l}\text { A. Autosuficiencia } \\
\text { alimentaria }\end{array}$} & \multirow{3}{*}{$\begin{array}{c}\text { B. } \\
\text { Ingreso } \\
\text { neto } \\
\text { mensual }\end{array}$} & \multicolumn{6}{|c|}{ C. Riesgo Económico } \\
\hline & $\mathbf{A 1}$ & $\mathbf{A 2}$ & & C1 & $\mathrm{C2}$ & $\mathbf{C 3}$ & $\mathrm{C4}$ & C5 & C6 \\
\hline & 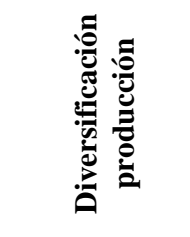 & 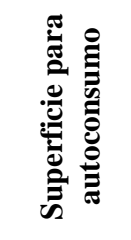 & & 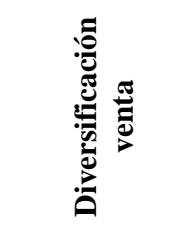 & 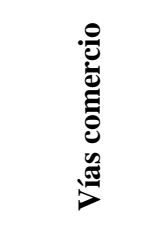 & 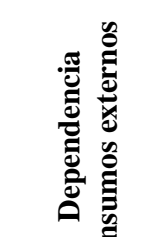 & 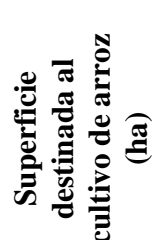 & 气 & 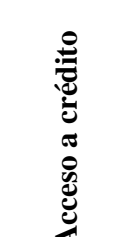 \\
\hline & (rubros) & (ha) & (dólares) & (rubros) & (canales) & & & & \\
\hline 4 & $\geq 5$ productos & $\geq 1$ & $\geq \operatorname{de} 384$ & $\geq 5$ productos & $\geq 5$ canales & 0 a $20 \%$ & $\geq 5$ & $\geq 7$ & $\begin{array}{l}\geq 4 \\
\text { fuentes } \\
\text { de } \\
\text { crédito }\end{array}$ \\
\hline 3 & 4 productos & $0.8-0.9$ & $300-383$ & 4 productos & 4 canales & 20 a $40 \%$ & 4 & $5-6$ & $\begin{array}{l}3 \\
\text { fuentes } \\
\text { de } \\
\text { crédito }\end{array}$ \\
\hline 2 & 3 productos & $0.5-0.7$ & $200-300$ & 3 productos & 3 canales & 40 a $60 \%$ & 3 & $4-5$ & $\begin{array}{l}2 \\
\text { fuentes } \\
\text { de } \\
\text { crédito }\end{array}$ \\
\hline 1 & 2 productos & $0.2-0.4$ & $100-200$ & 2 productos & 2 canales & 60 a $80 \%$ & 2 & $3-4$ & $\begin{array}{l}1 \text { fuente } \\
\text { de } \\
\text { crédito }\end{array}$ \\
\hline 0 & $\leq 1$ producto & $\leq 0.1$ & $\leq \operatorname{de} 100$ & $\leq 1$ producto & $\leq 1$ canal & 80 a $100 \%$ & $\leq 1$ & $\leq 3$ & $\begin{array}{l}\text { Sin } \\
\text { crédito }\end{array}$ \\
\hline
\end{tabular}

tn: tonelada métrica $\left(=10^{3} \mathrm{Kg}\right)$.

Fuente: Sarandón et al. (2006).

Tabla 4. Subindicadores y variables para evaluar el Indicador Ecológico (parte 1).

\begin{tabular}{|c|c|c|c|c|}
\hline \multirow[b]{2}{*}{ } & \multicolumn{2}{|c|}{ A. Conservación vida del suelo } & \multirow{2}{*}{$\begin{array}{c}\text { B- Manejo de la } \\
\text { Biodiversidad } \\
\text { B1 }\end{array}$} & \multirow{2}{*}{$\frac{\text { C. Plagas y enfermedades }}{\text { C1 }}$} \\
\hline & $\begin{array}{c}\text { A1 } \\
\text { Manejo } \\
\text { Cobertura } \\
\text { Vegetal } \\
(\%)\end{array}$ & Rotación de cultivos & & \\
\hline 4 & 100 & $\begin{array}{l}\text { Rota los cultivos } \\
\text { todos los años / Deja } \\
\text { descansar un año el } \\
\text { lote / incorpora } \\
\text { leguminosas o abonos } \\
\text { verdes. }\end{array}$ & $\begin{array}{l}\text { Establecimiento } \\
\text { totalmente } \\
\text { diversificado, con } \\
\text { asociaciones entre ellos } \\
\text { y con vegetación } \\
\text { natural. }\end{array}$ & $\begin{array}{l}\text { No se observan afectaciones por plagas, } \\
\text { enfermedades y arvenses. }\end{array}$ \\
\hline 3 & 75 a 99 & $\begin{array}{l}\text { Rota todos los años. } \\
\text { No deja descansar el } \\
\text { suelo. }\end{array}$ & $\begin{array}{l}\text { Alta diversificación de } \\
\text { cultivos, con media } \\
\text { asociación entre ellos. }\end{array}$ & $\begin{array}{l}\text { Afectaciones leves y autorregulables por el } \\
\text { sistema. }\end{array}$ \\
\hline 2 & 50 a 75 & Rota cada 2 - 3 años & $\begin{array}{l}\text { Diversificación media, } \\
\text { con muy bajo nivel de } \\
\text { asociación entre ellos. }\end{array}$ & $\begin{array}{l}\text { Afectaciones } 30-40 \% \text { de los cultivos, con } \\
\text { síntomas de leves y no hay arvenses } \\
\text { dominantes. }\end{array}$ \\
\hline 1 & 25 a 50 & $\begin{array}{l}\text { Realiza rotaciones } \\
\text { eventualmente }\end{array}$ & $\begin{array}{l}\text { Poca diversificación de } \\
\text { cultivos, sin } \\
\text { asociaciones. }\end{array}$ & $\begin{array}{l}\text { Afectaciones } 40-50 \% \text { de los cultivos, con } \\
\text { síntomas de leves a severos. }\end{array}$ \\
\hline 0 & $<25$ & No realiza rotaciones & Monocultivo & $\begin{array}{l}\text { Grandes afectaciones }>50 \% \text { plagas, } \\
\text { enfermedades en toda el área y presencia } \\
\text { de especies de arvenses dominantes. }\end{array}$ \\
\hline
\end{tabular}


Tabla 5. Subindicadores y variables para evaluar el Indicador Ecológico (parte 2).

C. Plagas y
enfermedades

C2

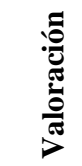

Aplicación de agroquímicos

-

D. Salinidad

D2

D3

\begin{tabular}{ccccc}
\hline 4 & $\leq 3$ & Valor de 1 & Valor de $<0.7$ & $(4)<100$ psi. 6.8 atmósferas \\
3 & 4 a 5 & Valor de 2 & Valor de 1 & (3) 100 a 150 psi 6.8 a 10.2 atmósferas \\
2 & 6 a 7 & Valor de 4 & Valor de 2 & (2) 150 a 200 psi 10.2 a 13.61 atmósferas \\
1 & 8 a 9 & Valor de 5 & Valor de 3 & (1) 200 a 250 psi. 13.61 a 17.0 atmósferas \\
0 & $\geq 10$ & Valor mayor a 5 & Valor $>$ a 3 & (0) $>300$ psi. 17.0 a 20.41 atmósferas \\
\hline \multicolumn{7}{l}{ CE: Conductividad Eléctrica; psi: Libras por pulgada cuadrada. Fuente: Sarandón et al. (2006). }
\end{tabular}

Tabla 6. Subindicadores y variables para evaluar la Dimensión Sociocultural.

\begin{tabular}{|c|c|c|c|c|c|c|c|}
\hline \multirow{2}{*}{ } & \multicolumn{4}{|c|}{$\begin{array}{c}\text { A. } \\
\text { Satisfacción necesidades básicas }\end{array}$} & \multirow{2}{*}{$\begin{array}{c}\text { B. } \\
\text { Aceptabilidad } \\
\text { sistema } \\
\text { producción. }\end{array}$} & \multirow{2}{*}{$\begin{array}{c}\text { C. } \\
\text { Integración } \\
\text { social }\end{array}$} & \multirow{2}{*}{$\begin{array}{c}\text { D. } \\
\text { Conocimiento } \\
\text { y conciencia } \\
\text { ecológica }\end{array}$} \\
\hline & $\begin{array}{c}\text { A1 } \\
\text { Vivienda }\end{array}$ & $\begin{array}{c}\text { A2 } \\
\text { Acceso a la } \\
\text { educación }\end{array}$ & $\begin{array}{c}\text { A3 } \\
\text { Acceso a salud } \\
\text { y cobertura } \\
\text { sanitaria } \\
\end{array}$ & $\begin{array}{c}\text { A4 } \\
\text { Servicios }\end{array}$ & & & \\
\hline 4 & $\begin{array}{l}\text { De material } \\
\text { noble, muy } \\
\text { buena. }\end{array}$ & $\begin{array}{c}\text { Acceso a } \\
\text { educación } \\
\text { superior y/o } \\
\text { cursos de } \\
\text { capacitación. }\end{array}$ & $\begin{array}{l}\text { Centro sanitario } \\
\text { con médicos } \\
\text { permanentes e } \\
\text { infraestructura } \\
\text { adecuada. }\end{array}$ & $\begin{array}{c}\text { Instalación } \\
\text { completa } \\
\text { de agua, } \\
\text { luz y } \\
\text { teléfono } \\
\text { cercano. }\end{array}$ & Muy satisfecha & Muy alta & $\begin{array}{c}\text { Con alta } \\
\text { conciencia } \\
\text { ecológica, } \\
\text { realiza } \\
\text { prácticas } \\
\text { conservacionist } \\
\text { as, no emplea } \\
\text { agroquímicos. }\end{array}$ \\
\hline 3 & $\begin{array}{l}\text { De material } \\
\text { noble, } \\
\text { buena. }\end{array}$ & $\begin{array}{c}\text { Acceso a } \\
\text { escuela } \\
\text { secundaria }\end{array}$ & $\begin{array}{c}\text { Centro sanitario } \\
\text { con personal } \\
\text { temporario } \\
\text { medianamente } \\
\text { equipado }\end{array}$ & $\begin{array}{c}\text { Instalación } \\
\text { de agua y } \\
\text { luz }\end{array}$ & Satisfecho & Alta & $\begin{array}{l}\text { Mediana } \\
\text { conciencia } \\
\text { ecológica }\end{array}$ \\
\hline 2 & $\begin{array}{c}\text { Regular, sin } \\
\text { terminar o } \\
\text { deteriorada }\end{array}$ & $\begin{array}{c}\text { Acceso a la } \\
\text { escuela primaria } \\
\text { y secundaria } \\
\text { con } \\
\text { restricciones }\end{array}$ & $\begin{array}{c}\text { Centro sanitario } \\
\text { mal equipado y } \\
\text { personal } \\
\text { temporario }\end{array}$ & $\begin{array}{c}\text { Instalación } \\
\text { de luz y } \\
\text { agua de } \\
\text { pozo }\end{array}$ & $\begin{array}{l}\text { Mediana } \\
\text { satisfacción }\end{array}$ & Media & $\begin{array}{l}\text { Visión } \\
\text { ecológica } \\
\text { reducida con } \\
\text { uso de algunos } \\
\text { agroquímicos. }\end{array}$ \\
\hline 1 & $\begin{array}{l}\text { Mala, sin } \\
\text { terminar, } \\
\text { deteriorada, } \\
\text { piso de } \\
\text { tierra }\end{array}$ & $\begin{array}{c}\text { Acceso a la } \\
\text { escuela primaria }\end{array}$ & $\begin{array}{c}\text { Centro sanitario } \\
\text { mal equipado y } \\
\text { sin personal } \\
\text { idóneo }\end{array}$ & $\begin{array}{l}\text { Sin } \\
\text { instalación } \\
\text { de luz y } \\
\text { agua de } \\
\text { pozo }\end{array}$ & $\begin{array}{c}\text { Poca } \\
\text { satisfacción }\end{array}$ & Baja & $\begin{array}{c}\text { No percibe } \\
\text { consecuencias. } \\
\text { Emplea } \\
\text { agroquímicos }\end{array}$ \\
\hline 0 & Muy mala & $\begin{array}{l}\text { Sin acceso a la } \\
\text { educación }\end{array}$ & $\begin{array}{l}\text { Sin centro } \\
\text { sanitario }\end{array}$ & $\begin{array}{l}\text { Sin Luz y } \\
\text { sin fuente } \\
\text { de agua } \\
\text { cercana }\end{array}$ & Desilusionado & Nula & $\begin{array}{l}\text { Sin conciencia } \\
\text { ecológica }\end{array}$ \\
\hline
\end{tabular}

Fuente: Sarandón et al. (2006). 


\begin{tabular}{|c|c|c|c|c|c|c|}
\hline CÓDIGO & CANTÓN & $\begin{array}{l}\text { TEXTURA DEL } \\
\text { SUELO }\end{array}$ & $\begin{array}{c}\% \\
\text { MO }\end{array}$ & $\begin{array}{c}\% \\
\text { ARENA }\end{array}$ & $\begin{array}{c}\% \\
\text { LIMO }\end{array}$ & $\begin{array}{c}\% \\
\text { ARCILLA }\end{array}$ \\
\hline P1 & Yaguachi & Arcilloso & 3.90 & 16 & 26 & 58 \\
\hline $\mathrm{P} 2$ & Yaguachi & Arcilloso & 2.42 & 14 & 26 & 60 \\
\hline P3 & Yaguachi & Arcilloso & 6.70 & 16 & 24 & 61 \\
\hline $\mathrm{P} 4$ & Yaguachi & Arcilloso & 3.01 & 12 & 48 & 40 \\
\hline P5 & Yaguachi & Arcillo-Limoso & 3.23 & 14 & 46 & 40 \\
\hline P6 & Yaguachi & Arcilloso & 2.46 & 18 & 33 & 49 \\
\hline P7 & Yaguachi & Arcilloso & 2.09 & 18 & 43 & 39 \\
\hline P8 & Yaguachi & Arcillo-Limoso & 4.08 & 14 & 45 & 41 \\
\hline P9 & Yaguachi & Arcilloso & 2.94 & 18 & 41 & 41 \\
\hline $\mathrm{P} 10$ & Yaguachi & Arcilloso & 4.37 & 12 & 34 & 54 \\
\hline P11 & Babahoyo & Arcilloso & 3.46 & 19 & 48 & 33 \\
\hline $\mathrm{P} 12$ & Babahoyo & Arcillo-Limoso & 3.08 & 20 & 50 & 30 \\
\hline P13 & Babahoyo & Arcillo-Limoso & 2.14 & 19 & 50 & 31 \\
\hline P14 & Babahoyo & Arcilloso & 2.44 & 21 & 48 & 31 \\
\hline $\mathrm{P} 15$ & Babahoyo & Arcilloso & 2.13 & 18 & 47 & 35 \\
\hline P16 & Babahoyo & Arcillo-Limoso & 2.73 & 16 & 50 & 34 \\
\hline P17 & Babahoyo & Arcilloso & 3.54 & 21 & 49 & 30 \\
\hline P18 & Babahoyo & Arcillo-Limoso & 2.14 & 19 & 50 & 31 \\
\hline P19 & Babahoyo & Arcilloso & 2.32 & 21 & 49 & 30 \\
\hline P20 & Babahoyo & Arcilloso & 3.4 & 19 & 49 & 32 \\
\hline
\end{tabular}

Fuente: Autores.

Tabla 8. Macro y microelementos de suelos muestreados en el cultivo del arroz en Yaguachi y Babahoyo.

\begin{tabular}{|c|c|c|c|c|c|c|c|c|c|c|c|}
\hline \multirow{3}{*}{ CÓDIGO } & \multirow{3}{*}{ CANTÓN } & \multicolumn{5}{|c|}{ MACROELEMENTOS } & \multirow{2}{*}{\multicolumn{5}{|c|}{$\begin{array}{c}\text { MICROELEMENTOS } \\
\text { mg/Kg (ppm) }\end{array}$}} \\
\hline & & \multirow{2}{*}{$\begin{array}{c}\text { mg/Kg } \\
\text { (ppm) } \\
P\end{array}$} & \multicolumn{2}{|c|}{ meq/100gr } & \multicolumn{2}{|c|}{$\begin{array}{c}\mathbf{m g} / \mathbf{K g} \\
(\mathbf{p p m})\end{array}$} & & & & & \\
\hline & & & $\mathbf{K}$ & $\mathbf{C a}$ & Mg & $\mathbf{S}$ & $\mathbf{Z n}$ & $\mathbf{C u}$ & Mn & $\mathbf{B}$ & $\mathbf{F e}$ \\
\hline P1 & Yaguachi & 4.21 & 0.416 & 19.0 & 16.0 & 7.24 & 4.55 & 9.78 & 51.6 & 0.69 & 32.6 \\
\hline $\mathrm{P} 2$ & Yaguachi & 6.98 & 0.488 & 19.1 & 20.3 & 7.45 & 1.11 & 10.3 & 12.2 & 0.58 & 9.9 \\
\hline P3 & Yaguachi & 7.78 & 0.388 & 18.5 & 12.9 & 6.34 & 2.31 & 21.8 & 44.4 & 0.91 & 34.1 \\
\hline P4 & Yaguachi & 10.2 & 0.22 & 14.9 & 4.5 & 7.90 & 3.20 & 10.12 & 21.1 & 0.98 & 193.0 \\
\hline P5 & Yaguachi & 16.4 & 0.13 & 16 & 5.78 & 7.21 & 3.30 & 16.8 & 140.3 & 1.13 & 174.4 \\
\hline P6 & Yaguachi & 13.9 & 0.751 & 20.1 & 16.4 & 12.20 & 1.74 & 8.08 & 19.8 & 0.82 & 8.3 \\
\hline P7 & Yaguachi & 37.5 & 0.19 & 14.33 & 3.35 & 7.90 & 3.10 & 12.2 & 140.0 & 0.98 & 253.3 \\
\hline P8 & Yaguachi & 24.98 & 0.37 & 20.29 & 11.4 & 7.21 & 3.70 & 10.7 & 94.0 & 0.87 & 14.2 \\
\hline P9 & Yaguachi & 97.3 & 1.58 & 23.01 & 12.7 & 7.81 & 15.80 & 10.7 & 94.0 & 1.32 & 14.2 \\
\hline P10 & Yaguachi & 3.00 & 0.284 & 15.9 & 10.3 & 12.90 & 1.02 & 25.0 & 128.5 & 0.38 & 89.2 \\
\hline P11 & Babahoyo & 24.67 & 0.19 & 16.11 & 3.8 & 6.90 & 3.40 & 13.1 & 169.2 & 1.01 & 190.5 \\
\hline P12 & Babahoyo & 11.73 & 0.15 & 12.85 & 4.18 & 7.65 & 2.40 & 21.3 & 247.4 & 0.96 & 404.2 \\
\hline P13 & Babahoyo & 30.2 & 0.12 & 18.39 & 3.22 & 6.45 & 2.30 & 11.9 & 81.4 & 1.05 & 86.6 \\
\hline P14 & Babahoyo & 14.5 & 0.15 & 15.65 & 5.15 & 5.83 & 2.10 & 17.6 & 92.4 & 0.98 & 168.2 \\
\hline P15 & Babahoyo & 10.08 & 0.17 & 15.69 & 5.14 & 5.70 & 1.90 & 12.2 & 144.8 & 0.90 & 123.1 \\
\hline P16 & Babahoyo & 11.7 & 0.1 & 15.8 & 4.67 & 6.71 & 3.10 & 9.7 & 78.2 & 0.97 & 81.7 \\
\hline P17 & Babahoyo & 3.9 & 0.09 & 18.09 & 5.41 & 6.41 & 1.40 & 20.9 & 94.5 & 1.00 & 116.2 \\
\hline P18 & Babahoyo & 25.2 & 0.12 & 18.22 & 3.22 & 6.45 & 3.30 & 12.5 & 82.0 & 1.05 & 85.3 \\
\hline P19 & Babahoyo & 6.6 & 0.12 & 15.62 & 5.93 & 5.62 & 1.40 & 15.7 & 167.9 & 1.07 & 122.9 \\
\hline $\mathrm{P} 20$ & Babahoyo & 4.42 & 0.15 & 20.45 & 9.85 & 8.31 & 1.90 & 23.6 & 93.7 & 1.24 & 31.5 \\
\hline
\end{tabular}


Enero - Julio 2021

Tabla 9. Valores de pH y CE de los suelos muestreados en el cultivo del arroz en Yaguachi y Babahoyo.

\begin{tabular}{lllcl}
\hline CÓDIGO & CANTÓN & pH & CE $(\mathbf{d S} / \mathbf{m})$ & \multicolumn{1}{c}{ Clasificación del suelo (FAO, 2012) } \\
\hline P1 & Yaguachi & 6.53 & 9.3 & Fuertemente salinos \\
P2 & Yaguachi & 7.51 & 5.0 & Salinos \\
P3 & Yaguachi & 7.28 & 6.8 & Salinos \\
P4 & Yaguachi & 6.92 & 5.4 & Salinos \\
P5 & Yaguachi & 5.32 & 6.5 & Salinos \\
P6 & Yaguachi & 7.20 & 7.0 & Salinos \\
P7 & Yaguachi & 6.25 & 4.2 & Salinos \\
P8 & Yaguachi & 6.94 & 9.4 & Fuertemente salinos \\
P9 & Yaguachi & 7.02 & 9.2 & Fuertemente salinos \\
P10 & Yaguachi & 7.49 & 7.4 & Salinos \\
P11 & Babahoyo & 6.24 & 1.1 & Normales \\
P12 & Babahoyo & 5.39 & 1.8 & Normales \\
P13 & Babahoyo & 6.12 & 1.9 & Normales \\
P14 & Babahoyo & 6 & 2.0 & Normales \\
P15 & Babahoyo & 5.99 & 1.7 & Normales \\
P16 & Babahoyo & 6.6 & 1.1 & Normales \\
P17 & Babahoyo & 5.73 & 2.7 & Ligeramente salinos \\
P18 & Babahoyo & 6.12 & 0.3 & Normales \\
P19 & Babahoyo & 6.05 & 1.8 & Normales \\
P20 & Babahoyo & 6.32 & 2.2 & Ligeramente salinos \\
\hline
\end{tabular}

CE: Conductividad Eléctrica. Fuente: Autores.

Tabla 10. Valores de compactación (psi) de los suelos muestreados en el cultivo del arroz en Yaguachi y Babahoyo.

\begin{tabular}{llll}
\hline CÓDIGO & CANTÓN & psi & Condición \\
\hline P1 & Yaguachi & 300 & Malo para cultivo \\
P2 & Yaguachi & 200 & Regular para el cultivo \\
P3 & Yaguachi & 230 & Regular para el cultivo \\
P4 & Yaguachi & 200 & Regular para el cultivo \\
P5 & Yaguachi & 230 & Regular para el cultivo \\
P6 & Yaguachi & 260 & Regular para el cultivo \\
P7 & Yaguachi & 180 & Bueno para cultivo \\
P8 & Yaguachi & 250 & Regular para el cultivo \\
P9 & Yaguachi & 270 & Regular para el cultivo \\
P10 & Yaguachi & 230 & Regular para el cultivo \\
P11 & Babahoyo & 150 & Bueno para cultivo \\
P12 & Babahoyo & 160 & Bueno para cultivo \\
P13 & Babahoyo & 150 & Bueno para cultivo \\
P14 & Babahoyo & 150 & Bueno para cultivo \\
P15 & Babahoyo & 150 & Bueno para cultivo \\
P16 & Babahoyo & 120 & Bueno para cultivo \\
P17 & Babahoyo & 200 & Regular para el cultivo \\
P18 & Babahoyo & 100 & Bueno para cultivo \\
P19 & Babahoyo & 150 & Bueno para cultivo \\
P20 & Babahoyo & 180 & Bueno para cultivo \\
\hline
\end{tabular}

Fuente: Autores.
Tabla 11. Valores de $\mathrm{pH}$ y CE de las muestras de las aguas colectadas en el cultivo del arroz en Babahoyo.

\begin{tabular}{lllc}
\hline CÓDIGO & CANTÓN & pH & CE $(\mathbf{d S} / \mathbf{m})$ \\
\hline P11 & Babahoyo & 7.1 & 0.5 \\
P12 & Babahoyo & 7.2 & 0.2 \\
P13 & Babahoyo & 7.1 & 0.1 \\
P14 & Babahoyo & 7.2 & 0.5 \\
P15 & Babahoyo & 7.9 & 0.6 \\
P16 & Babahoyo & 6.6 & 0.2 \\
P17 & Babahoyo & 7.5 & 0.9 \\
P18 & Babahoyo & 7.6 & 0.2 \\
P19 & Babahoyo & 7.5 & 0.3 \\
P20 & Babahoyo & 7.6 & 0.7 \\
\hline
\end{tabular}

CE: Conductividad eléctrica. Fuente: Autores 
Tabla 12. Descripción de las propiedades físico-químicas del agua de riego de Yaguachi.

\begin{tabular}{|c|c|c|c|c|c|c|c|c|c|c|c|c|}
\hline \multirow[t]{2}{*}{ CÓDIGO } & \multirow[t]{2}{*}{ pH } & \multirow{2}{*}{$\frac{\mathrm{dS} / \mathrm{m}}{\mathrm{CE}}$} & \multirow{2}{*}{$\begin{array}{l}\mathrm{mg} / \mathrm{l} \\
\text { STD }\end{array}$} & \multicolumn{9}{|c|}{ ppm (mg/l) } \\
\hline & & & & $\mathbf{N a}$ & $\mathbf{K}$ & $\mathrm{Ca}$ & Mg & $\begin{array}{l}\text { Dureza } \\
\text { cálcica } \\
\mathrm{CaCO}_{3}\end{array}$ & $\begin{array}{c}\text { Dureza } \\
\text { Magnésica } \\
\mathrm{MgCO}_{3}\end{array}$ & $\begin{array}{c}\text { Dureza } \\
\text { Total }\end{array}$ & $\mathrm{Cl}^{-}$ & RAS \\
\hline $\mathrm{P} 1$ & 6.91 & 2.49 & 1350 & 479.4 & 26.3 & 55.3 & 75.6 & 138.2 & 262.1 & 400.3 & 689.4 & 9.8 \\
\hline $\mathrm{P} 2$ & 6.44 & 2.84 & 1520 & 514.8 & 23.7 & 25.0 & 58.3 & 62.3 & 202.1 & 264.4 & 713.5 & 12.9 \\
\hline P3 & 6.78 & 2.53 & 1360 & 442.6 & 20.6 & 49.7 & 68.2 & 124.1 & 236.4 & 360.5 & 675.0 & 9.6 \\
\hline P4 & 6.70 & 2.63 & 1440 & 485.6 & 19.8 & 57.3 & 75.1 & 143.2 & 260.4 & 403.6 & 723.2 & 9.9 \\
\hline P5 & 6.77 & 2.74 & 1470 & 633.5 & 10.1 & 128.3 & 115.3 & 320.4 & 399.9 & 720.3 & 1026.9 & 9.8 \\
\hline P6 & 6.90 & 3.52 & 1920 & 489.7 & 19.6 & 29.4 & 71.4 & 73.3 & 247.5 & 320.8 & 935.3 & 11.1 \\
\hline P7 & 7.01 & 2.32 & 1240 & 449.5 & 17.5 & 21.8 & 52.0 & 54.3 & 180.5 & 234.8 & 660.7 & 11.9 \\
\hline P8 & 6.90 & 2.810 & 1580 & 191.1 & 7.4 & 93.4 & 69.6 & 233.1 & 241.3 & 474.4 & 626.8 & 3.65 \\
\hline P9 & 7.06 & 2.460 & 1330 & 171.1 & 6.6 & 80.60 & 63.39 & 201.0 & 219.9 & 421.0 & 761.7 & 3.46 \\
\hline $\mathrm{P} 10$ & 7.16 & 2.090 & 1120 & 152.0 & 8.0 & 64.6 & 51.7 & 161.3 & 179.3 & 340.7 & 429.1 & 3.42 \\
\hline
\end{tabular}

Tabla 13. Resumen de los resultados de la caracterización de fincas arroceras, dimensión económica.

\begin{tabular}{|c|c|}
\hline Indicadores & Resultados \\
\hline $\begin{array}{l}\text { Diversificación de } \\
\text { la producción }\end{array}$ & $\begin{array}{l}\text { Los productores arroceros de Yaguachi y Babahoyo en su mayoría mantuvieron un sistema de } \\
\text { monocultivo (arroz) con } 42 \text { y } 52 \% \text {, respectivamente, lo cual genera pérdidas de ingresos } \\
\text { adicionales y la falta de diversidad de productos que puedan ser utilizados para la alimentación. } \\
\text { Por otro lado, existe otro grupo de productores con } 33 \text { y } 34 \% \text {; respectivamente, que mantiene en } \\
\text { sus predios otros cultivos como son: plátano, cacao y yuca, que generalmente son para la } \\
\text { alimentación del grupo familiar y una parte para la comercialización (Figura } 2 \text { ). }\end{array}$ \\
\hline $\begin{array}{l}\text { Superficie de } \\
\text { producción } \\
\text { destinada al } \\
\text { autoconsumo }\end{array}$ & $\begin{array}{l}\text { Del total de la superficie para autoconsumo, un gran porcentaje de productores en Yaguachi } \\
\text { destinaron de } 0.2-0.4 \text { ha, lo cual representó el } 60 \% \text {. Con un resultado similar se encontraron los } \\
\text { productores de Babahoyo, los cuales dedicaron de } 0.2-0.4 \text { ha para su autoconsumo, con un valor } \\
\text { de } 59 \% \text { (Figura } 3 \text { ). }\end{array}$ \\
\hline $\begin{array}{l}\text { Diversificación } \\
\text { para la venta }\end{array}$ & $\begin{array}{l}\text { El } 47 \% \text { de los productores de Yaguachi ofertaron al mercado un producto (arroz); Babahoyo con } \\
59 \% \text {. Estos resultados muestran que más de la mitad del área es monocultivo (Figura 4). }\end{array}$ \\
\hline Ingresos & $\begin{array}{l}\text { Para el 55\% de los productores de arroz de Yaguachi (Figura 4) y el 58\% de los productores de } \\
\text { arroz de Babahoyo, el ingreso neto mensual en el } 2018 \text { superó los } 384 \text { USD (salario mínimo en } \\
\text { el año } 2019 \text { en Ecuador) (Figura 5). }\end{array}$ \\
\hline $\begin{array}{l}\text { Vías de } \\
\text { comercialización }\end{array}$ & $\begin{array}{l}\text { El } 48 \% \text { de los arroceros de Yaguachi y el } 60 \% \text { de los productores de Babahoyo utilizaron un } \\
\text { solo canal de comercialización (Figura } 6 \text { ). }\end{array}$ \\
\hline $\begin{array}{l}\text { Dependencia de } \\
\text { insumos externos }\end{array}$ & $\begin{array}{l}\text { El 56\% de los productores de Yaguachi y el } 63 \% \text { de los arroceros de Babahoyo se consideraron } \\
\text { dependientes de insumos externos de un } 60 \% \text { y } 80 \% \text { de estos, respectivamente (Figura } 7 \text { ). }\end{array}$ \\
\hline $\begin{array}{l}\text { Productividad / } \\
\text { área }\end{array}$ & $\begin{array}{l}\text { En la zona de Yaguachi y Babahoyo, el } 35 \% \text { y } 39 \% \text { de los productores arroceros, el mayor } \\
\text { porcentaje está concentrado en el rango de } 4-5 \text { t, respectivamente (Figuras } 8 \text { ). }\end{array}$ \\
\hline Fuentes de crédito & $\begin{array}{l}\text { El } 70 \% \text { de los arroceros de Yaguachi y el } 66 \% \text { de los de Babahoyo, carecían de líneas de crédito } \\
\text { (Figura 9). }\end{array}$ \\
\hline
\end{tabular}

Tabla 14. Resumen de los resultados de la caracterización de fincas arroceras, dimensión ecológica.

\begin{tabular}{|c|c|}
\hline Indicadores & Resultados \\
\hline $\begin{array}{l}\text { Manejo de la } \\
\text { cobertura vegetal }\end{array}$ & $\begin{array}{l}\text { El } 90 \% \text { y } 48 \% \text { de los productores de Yaguachi y Babahoyo, respetivamente, } \\
\text { mantuvieron en promedio un } 25 \% \text { de cobertura vegetal en el borde de los canales } \\
\text { y en pequeñas áreas colindantes con cultivos como yuca, plátano, cacao (Figura } \\
\text { 10). }\end{array}$ \\
\hline $\begin{array}{l}\text { Rotación de } \\
\text { cultivos }\end{array}$ & $\begin{array}{l}\text { El } 36 \% \text { de los productores de Yaguachi rotaron cada } 2 \text { a } 3 \text { años con cultivos de } \\
\text { ciclo corto, un } 27 \% \text { rotaron todos los años pero no dejaron descansar el suelo, y } \\
\text { un } 18 \% \text { no realizaron rotaciones. Por otro lado, en Babahoyo las proporciones } \\
\text { fueron de } 33 \%, 22 \% \text { y } 13 \% \text {, respectivamente (Figura } 11 \text { ). }\end{array}$ \\
\hline $\begin{array}{l}\text { Incidencia de } \\
\text { plagas }\end{array}$ & $\begin{array}{l}\text { El } 48 \% \text { de los productores de Yaguachi señalaron problemas de plagas y } \\
\text { enfermedades del arroz de entre } 30-40 \% \text {. El } 55 \% \text { de los arroceros de Babahoyo } \\
\text { presentaron daños de entre } 30 \text { a } 40 \% \text { por los mismos problemas (Figura 12). }\end{array}$ \\
\hline $\begin{array}{l}\text { Aplicación de } \\
\text { agroquímicos }\end{array}$ & $\begin{array}{l}\text { El } 62 \% \text { de los productores de Yaguachi y el } 33 \% \text { de Babahoyo realizaron entre } 8 \\
-9 \text { aplicaciones de agroquímicos (Figura 13). }\end{array}$ \\
\hline
\end{tabular}


Enero - Julio 2021

Tabla 15. Resumen de los resultados de la caracterización de fincas arroceras, dimensión sociocultural.

\begin{tabular}{|c|c|}
\hline Indicadores & Resultados \\
\hline Vivienda & $\begin{array}{l}\text { El } 79 \% \text { y el 59\% de los productores de Yaguachi y Babahoyo, tuvieron viviendas con buenas } \\
\text { características, respectivamente. }\end{array}$ \\
\hline Acceso a la educación & $\begin{array}{l}\text { Tuvieron acceso a colegios y capacitación el } 37 \text { y } 39 \% \text { de los productores de Yaguachi y } \\
\text { Babahoyo, respectivamente. Además, contaron con educación superior el } 18 \% \text { y } 23 \% \text { de } \\
\text { productores de Yaguachi y Babahoyo, respectivamente (Figura 14). }\end{array}$ \\
\hline $\begin{array}{l}\text { Acceso a salud y } \\
\text { cobertura sanitaria }\end{array}$ & $\begin{array}{l}\text { El } 40 \% \text { y el } 30 \% \text { de los productores de Yaguachi y Babahoyo, respectivamente, manifestaron } \\
\text { tener un centro sanitario con personal temporario medianamente equipado, y solo el } 16 \% \text { y } 13 \% \\
\text { de los encuestados en las dos zonas de estudio, respectivamente, cuentan con un centro sanitario } \\
\text { con médicos permanentes e infraestructura adecuada (Figura 15). }\end{array}$ \\
\hline Servicios & $\begin{array}{l}\text { Igualmente, en los dos sistemas analizados para la variable de servicios básicos, el } 72 \% \text { y el } 71 \% \\
\text { de los arroceros de Yaguachi y Babahoyo, respectivamente, cuentan con instalación de luz y } \\
\text { agua de pozo. Un } 5 \% \text { y } 7 \% \text { cuenta con instalación completa de agua, luz y teléfono, } \\
\text { respectivamente. }\end{array}$ \\
\hline $\begin{array}{l}\text { Aceptabilidad del } \\
\text { sistema de producción }\end{array}$ & $\begin{array}{l}\text { Del total de agricultores encuestados sobre el sistema de producción agrícola arrocera que } \\
\text { realizan como un medio de subsistencia, el } 48 \% \text { y el } 43 \% \text { de Yaguachi y Babahoyo, } \\
\text { respectivamente, manifestaron estar satisfechos. En el mismo orden, el } 12 \% \text { y el } 26 \% \text { de los } \\
\text { productores, de las zonas de estudio, señalaron no estar satisfechos y siguen en el sistema de } \\
\text { producción de arroz por ser la única actividad que conocen (Figura 16). }\end{array}$ \\
\hline Integración social & $\begin{array}{l}\text { El } 66 \% \text { y } 54 \% \text { de los agricultores de Yaguachi y Babahoyo, respectivamente, consideraron muy } \\
\text { alta la integración y relaciones con sus vecinos y otros agricultores. }\end{array}$ \\
\hline $\begin{array}{l}\text { Conocimiento y } \\
\text { conciencia ecológica }\end{array}$ & $\begin{array}{l}\text { El } 8 \% \text { y el } 16 \% \text { de los agricultores de Yaguachi y Babahoyo, respectivamente, manifestaron } \\
\text { tener conocimiento de ecología y sus fundamentos (Figura 17). }\end{array}$ \\
\hline
\end{tabular}

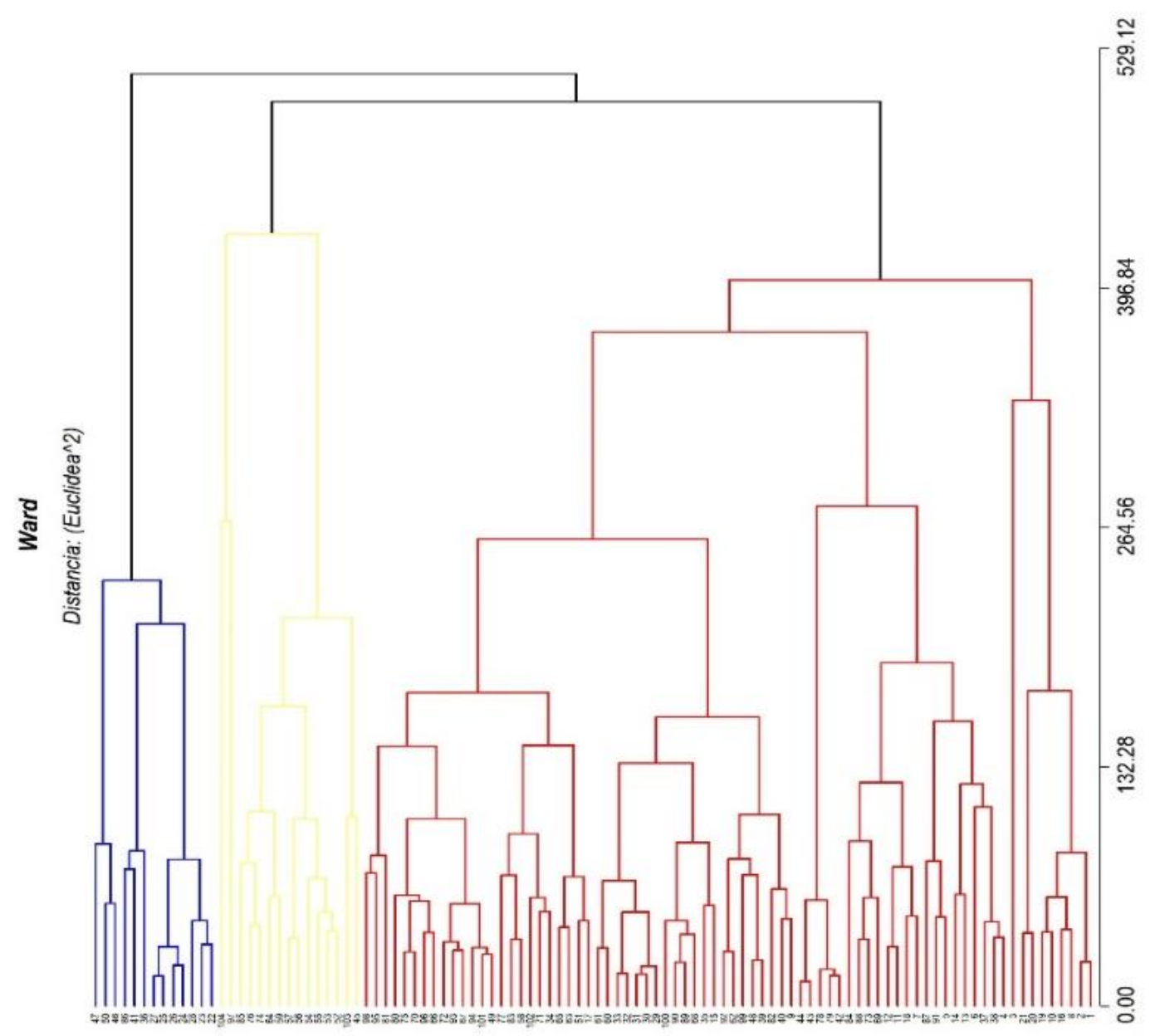

Figura 18. Tipificación de fincas arroceras en Babahoyo. 


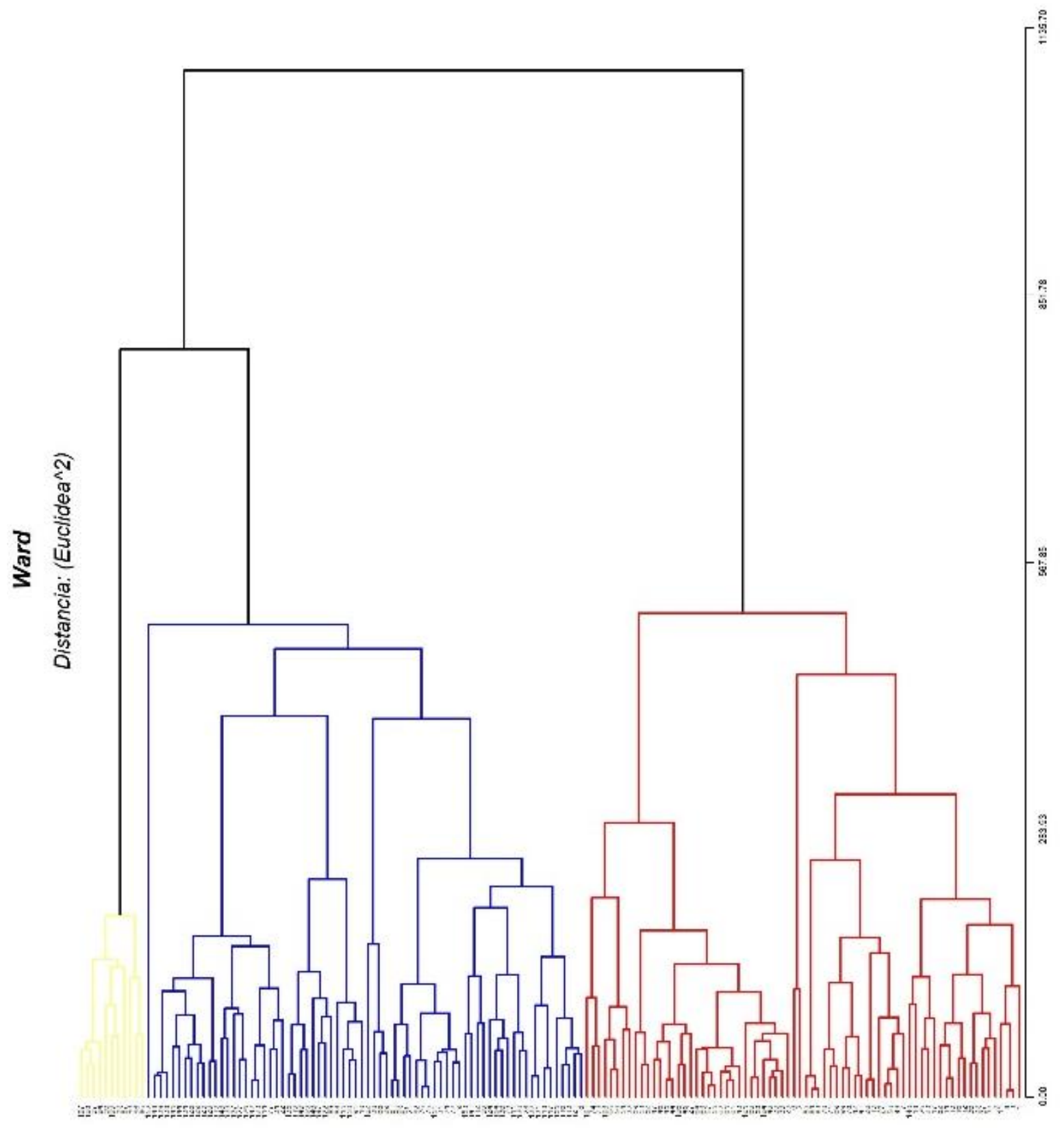

Figura 19. Tipificación de fincas arroceras en Yaguachi.

${ }^{1}$ Docente. Facultad de Ciencias Agropecuarias, Universidad Técnica de Babahoyo. Babahoyo, Ecuador. fcobos@utb.ec.

${ }^{2}$ Docente. Departamento de Fitotecnia, Facultad de Agronomía, Universidad Nacional Agraria La Molina, Lima, Perú. luzgomez@lamolina.edu.pe.

${ }^{3}$ Docente. Facultad de Ciencias Agropecuarias, Universidad Técnica de Babahoyo. Babahoyo, Ecuador. wreyes@utb.ec.

${ }^{4}$ Docente. Facultad de Ciencias Agrarias, Universidad de Guayaquil. Guayaquil, Ecuador. reina.medinal@ug.edu.ec. 